\title{
Mathematical Model for Low Density Lipoprotein (LDL) Endocytosis by Hepatocytes
}

\author{
J.A.D. Wattis ${ }^{\mathrm{a}, *}$, B. O’Malley ${ }^{\mathrm{b}}$, H. Blackburn ${ }^{\mathrm{b}}$, L. Pickersgill ${ }^{\mathrm{b}}$, J. Panovska ${ }^{\mathrm{b}}$, \\ H.M. Byrne ${ }^{\mathrm{a}}$, K.G. Jackson ${ }^{\mathrm{c}}$ \\ ${ }^{a}$ Centre for Mathematical Medicine and Biology, School of Mathematical Sciences, \\ University of Nottingham, University Park, Nottingham, NG7 2RD, UK \\ ${ }^{b}$ Unilever Corporate Research-Biosciences, Unilever Corporate Research, Colworth Park, \\ Sharnbrook, MK44 1LQ, UK \\ ${ }^{c}$ Hugh Sinclair Unit of Human Nutrition, Department of Food Biosciences, University of \\ Reading, Reading RG6 6AP, UK
}

Received: 28 March 2008 / Accepted: 16 July 2008 / Published online: 21 August 2008 (C) The Author(s) 2008. This article is published with open access at Springerlink.com

\begin{abstract}
Individuals with elevated levels of plasma low density lipoprotein (LDL) cholesterol (LDL-C) are considered to be at risk of developing coronary heart disease. LDL particles are removed from the blood by a process known as receptor-mediated endocytosis, which occurs mainly in the liver. A series of classical experiments delineated the major steps in the endocytotic process; apolipoprotein B-100 present on LDL particles binds to a specific receptor (LDL receptor, LDL-R) in specialized areas of the cell surface called clathrin-coated pits. The pit comprising the LDL-LDL-R complex is internalized forming a cytoplasmic endosome. Fusion of the endosome with a lysosome leads to degradation of the LDL into its constituent parts (that is, cholesterol, fatty acids, and amino acids), which are released for reuse by the cell, or are excreted.

In this paper, we formulate a mathematical model of LDL endocytosis, consisting of a system of ordinary differential equations. We validate our model against existing in vitro experimental data, and we use it to explore differences in system behavior when a single bolus of extracellular LDL is supplied to cells, compared to when a continuous supply of LDL particles is available. Whereas the former situation is common to in vitro experimental systems, the latter better reflects the in vivo situation. We use asymptotic analysis and numerical simulations to study the longtime behavior of model solutions. The implications of model-derived insights for experimental design are discussed.
\end{abstract}

Keywords Endocytosis · LDL uptake · Regulation of LDL-receptor production · PCSK9

\footnotetext{
* Corresponding author.

E-mail addresses: Jonathan.Wattis@ nottingham.ac.uk (J.A.D. Wattis), Brendan.O’Malley@unilever.com (B. O’Malley), Helen.Byrne@ nottingham.ac.uk (H.M. Byrne).
} 


\section{Introduction}

Complex biological mechanisms have evolved to transport cholesterol around the body, and to prevent accumulation of toxic levels of cholesterol within cells. A family of macromolecular complexes, known as lipoproteins, transport cholesterol through the bloodstream to the major tissues. In humans, low density lipoprotein (LDL) particles carry the majority of the plasma cholesterol, and elevated levels of LDL cholesterol (LDL-C) is a widely accepted risk factor for the development of coronary heart disease (CHD). Advances in our understanding of LDL metabolism have led to the identification of lifestyle or pharmaceutical interventions which improve plasma LDL-C levels; however, raised levels of plasma LDL-C remain a concern for human health, and more effective interventions are required.

LDL-C levels are in part determined by the rate at which LDL particles are taken up and removed from the circulation. The liver cells (hepatocytes) are responsible for the major part of whole body LDL uptake. The process by which LDL is taken up and processed by hepatocytes is known as receptor mediated endocytosis and involves a sequence of well-orchestrated mechanisms, which have been well defined in a series of classical experiments by Brown and Goldstein (1979). The first step in this process involves an LDL particle binding to hepatic LDL receptors (LDL-R) in specialized regions of the cell surface known as clathrin-coated pits. The interaction with the LDL-R is mediated by LDL-associated apolipoprotein B (apo B-100). Upon binding to the LDL-R, LDL particles become internalized into the cell, forming intracellular vesicles known as endosomes. In this paper, we use the term internalized pits to refer to endosomes.

Fusion of endosomes with lysosomes results in degradation of the LDL particles, and the release of its constituent parts (e.g., cholesterol, fatty acids, and amino acids). The LDL-Rs may be either recycled prior to lysosomal fusion, or degraded. A negative feedback mechanism regulates the number of cell surface LDL-R on the basis of the levels of intracellular free cholesterol, such that when they are elevated the number of LDL-Rs is reduced, whereas when they are low, the number of LDL-Rs is increased.

In vitro assays are widely used to study LDL cellular metabolism (Bradley et al., 1984; Brown and Goldstein, 1979; Cho et al., 2002; Jackson et al., 2005, 2006; Mamotte et al., 1999). These assays, which quantify the rate of LDL uptake by cultured cells, are used to investigate the steps of endocytosis, and to explore the mechanisms underlying the reduced rates of LDL uptake exhibited under specific experimental conditions. The assays typically involve adding an amount of lipoprotein spiked with radiolabeled LDL to the cell culture medium at a fixed timepoint, and tracking the movement of radiolabeled LDL into the cell over time. In vivo, rather than being exposed to a single bolus of lipoprotein, cells are typically exposed to a continuous source of lipoproteins, an effect which is technically challenging to reproduce in vitro. A major focus of this paper, is to assess the effect that such differences in the rate of lipoprotein delivery, has on the system behavior, and to identify methods to improve experimental design.

Over the past 20 years, a number of models of LDL metabolism have been formulated, which vary in both the mathematical approaches taken and biological scope, see August et al. (2007), Chun et al. (1985), Harwood and Pellarin (1997), Shankaran et al. (2007), for example. In several of these models, certain parameters were fitted in order to achieve agreement with experimental data from cultured human fibroblasts. Dynamic models have 
also been formulated. These tend to focus on specific areas of LDL metabolism, for example, fluid dynamics of lipid accumulation in the arterial wall, or the effect of antioxidants on the kinetics of LDL oxidation. August et al. (2007) developed a dynamic model of the lipoprotein delipidation cascade, consisting of a system of nonlinear differential equations which are more strongly linked to the underlying physiological processes. The model links lipoprotein metabolism at a physiological level, to the cellular level, by including a feedback mechanism whereby the cell regulates the amount of LDL receptors available for lipoprotein binding in response to the intracellular cholesterol concentration.

The model described in Section 2 of this paper is similar in form to that developed by August et al. (2007), that is, a system of ordinary differential equations (ODEs). However, rather than addressing the kinetics of the lipoprotein delipidation cascade, our model focuses in greater detail on the uptake of LDL by hepatocytes in culture, and incorporates greater detail on the endocytotic process than included in the model in August et al. (2007). In Section 2, we also estimate the model parameters and nondimensionalize the governing equations. In Section 3, we present typical numerical results and compare them to experimental data before performing a parameter sensitivity analysis. We confirm the numerical results and perform a steady state analysis to investigate the system's long term behavior in more detail in Section 4. Finally, in Section 5, we discuss the results. Our focus throughout the paper is on the endosome dynamics and the assumption that concentrations and fluxes are modulated by intracellular cholesterol levels.

\section{Model development}

In this section, we formulate the mathematical model that we use to study the dynamics of LDL endocytosis by hepatocytes or HepG2 cells, which is a cell line derived from a patient with hepatocellular carcinoma. We construct a model based on a system of ordinary differential equations which describe the evolution of spatially-averaged concentrations of LDL and cholesterol. Whilst the concentration of LDL in bulk extracellular medium may not be uniform, we will assume that there is a layer of fluid close to the cells in which the spatially-averaged concentration of LDL particles can be defined. The reason for this is our desire to focus on the dynamics of adhesion, internalization, and receptor-regulation rather than the fluid dynamics of LDL-delivery to the cell surface.

We model the transformation of a pit on the cell surface from being free of LDL to being bound with a certain number of LDL particles. By considering the density of pits bound with $0,1, \ldots$ up to a maximum of $p_{m}$ LDL particles, we construct a system of a large number of ordinary differential equations (ODES) (specifically, a system of size $\left.p_{m}+1,0<p_{m}<\infty\right)$, that enable us to monitor how the total number of pits per unit volume and their occupancy change over time. By a judicious choice of parameter values, we then show how to reduce the model to one which requires only three quantities to describe the attachment of LDL particles to the coated pits: the concentration of pits either containing, or completely free of, bound LDL particles $\left(\bar{N}, \bar{N}_{0}\right.$, respectively), and the concentration of LDL bound $(\bar{M})$. The model also describes the evolution of the concentration of LDL particles in the extracellular medium $\left(\bar{L}_{e}\right)$, as well as the changes in concentration of bound $\left(\bar{L}_{b}\right)$ and internalized $\left(\bar{L}_{i}\right)$ LDL particles and intracellular LDLderived cholesterol $(\bar{C})$. The processes are summarized in Fig. 1. 


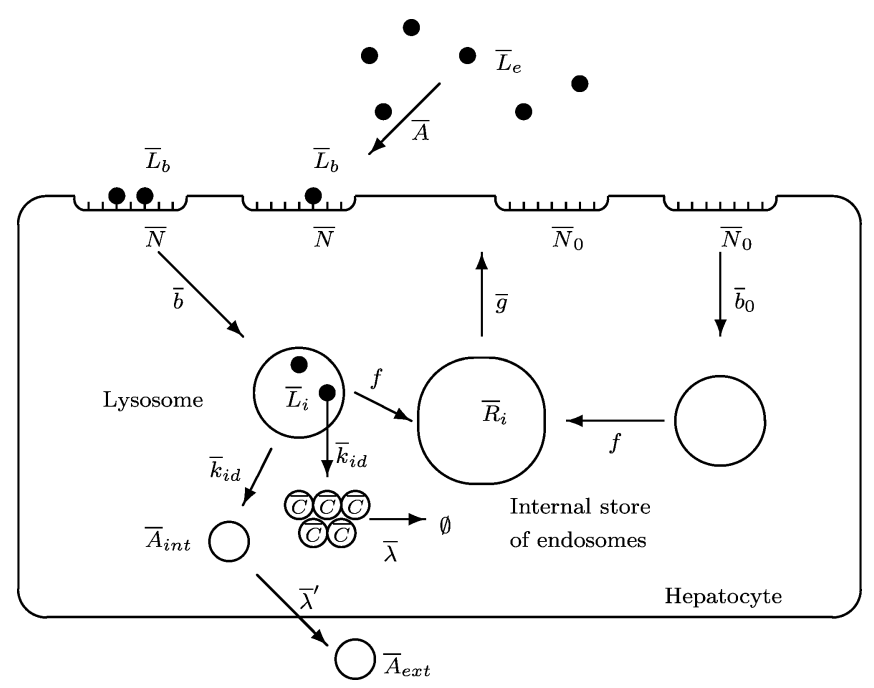

Fig. 1 Pictorial view of endocytosis in HepG2 cells. The parameters $\bar{A}, \bar{b}, \bar{b}_{0}$, and $\bar{g}$ are dimensional rate constants for the processes of LDL-binding to pit receptors, occupied, and empty pit (receptor) internalization, and pit recycling (see the main text).

\subsection{Microscopic modeling of pit dynamics}

We denote by $\bar{N}_{p}(\bar{t})$ the concentration of pits with $p$ LDL particles bound, $p$ being in the range $0 \leq p \leq p_{m}$, and the concentration being measured in numbers per unit volume. Then $\bar{N}_{0}(\bar{t})$ represents the number of pits per unit volume that are free of LDL at time $\bar{t}$ and $p_{m}$ denotes the maximum number of LDL particles that can bind in an individual coated pit $\left(0<p_{m}<\infty\right)$. In developing our model, we start by considering how $\bar{N}_{0}(\bar{t})$ evolves. We assume that empty pits are produced at a rate $\bar{k}_{0}$. LDL may bind to the empty pits, and once the first LDL particle is bound to a pit, more LDL particles may bind within a given pit, provided it is not full. We assume that time can be split into consecutive intervals, all small enough that at most only one binding event occurs in any interval. This means we only have to consider how $\bar{N}_{p}$ is related to $\bar{N}_{p-1}$, and we can ignore any direct dependence on $\bar{N}_{p-2}, \bar{N}_{p-3}$ etc. We define the sequential binding of LDL particles at a rate $\bar{a}_{p}$ (which depends on the current occupancy of the pit) by the iterative process

$$
N_{p-1}+L_{e} \stackrel{\bar{a}_{p-1}}{\longrightarrow} N_{p}+L_{b} \quad\left(1 \leq p \leq p_{m}\right)
$$

where $N_{p}$ denotes a pit with $p$ LDL particles attached, $L_{e}, L_{b}$ denote LDL particles in the extracellular space and bound to the pit, respectively. We assume that pits are internalized at a rate $\bar{b}$ if occupied and a different rate, $\bar{b}_{0}$, if empty. The equations for $\bar{N}_{p}(t)$, which are the time-dependent concentrations $\left[\bar{N}_{p}\right]$ for $p=0,1, \ldots, p_{m}$, have a source term due to LDL binding at rate $\bar{a}_{p-1}$ to a pit with $(p-1)$ LDL particles $\left(\bar{N}_{p-1}\right)$, and two sink terms: one due to the binding of LDL particles, and another due to internalization at a 
rate $\bar{b}$. Combining these mechanisms, we have

$$
\begin{aligned}
\frac{\mathrm{d} \bar{N}_{0}}{\mathrm{~d} \bar{t}} & =\bar{k}_{0}-\bar{a}_{0} \bar{L}_{e} \bar{N}_{0}-\bar{b}_{0} \bar{N}_{0} \\
\frac{\mathrm{d} \bar{N}_{p}}{\mathrm{~d} \bar{t}} & =\bar{a}_{p-1} \bar{L}_{e} \bar{N}_{p-1}-\bar{b} \bar{N}_{p}-\bar{a}_{p} \bar{L}_{e} \bar{N}_{p} \quad\left(1 \leq p \leq p_{m}-1\right), \\
\frac{\mathrm{d} \bar{N}_{p_{m}}}{\mathrm{~d} \bar{t}} & =\bar{a}_{p_{m-1}-1} \bar{L}_{e} \bar{N}_{p_{m-1}}-\bar{b} \bar{N}_{p_{m}}
\end{aligned}
$$

where the production rate $\bar{k}_{0}$ is due to the transport of receptors from internal stores to the cell surface. To account for this process, we introduce a new variable, $\bar{R}_{i}$ which represents the number of pits per unit volume in the internal store. Pits in this store arise from two different sources. Firstly, we assume that a fraction $f$ (typically 70\%-100\% (Dunn et al., 1989)) of internalized pits enter the store. New pits are also produced within the cell at a rate which increases as the intracellular cholesterol level falls (Dunn et al., 1989). Finally, pits are released from the store to the cell surface at a constant rate $\bar{g}$ (Dunn et al., 1989). Combining these effects, we obtain the equation

$$
\frac{\mathrm{d} \bar{R}_{i}}{\mathrm{~d} \bar{t}}=\frac{\bar{k}_{s}}{\bar{K}+\bar{C}}+f \bar{b} \sum_{p=1}^{p_{m}} \bar{N}_{p}+f \bar{b}_{0} \bar{N}_{0}-\bar{g} \bar{R}_{i} .
$$

We note that as a result of introducing $\bar{R}_{i}$ we now have $\bar{k}_{0}=\bar{g} \bar{R}_{i}$ in (2). In Eq. (5), $\bar{k}_{s}$ and $\bar{K}$ are constants which describe how the cells regulate de novo synthesis of pits. This gives a lower pit production rate when the cell's internal cholesterol level is high and a higher production rate when $\bar{C}$ is low. The pit production rate also depends on the concentration of pits in the store $\bar{R}_{i}$, which introduces some history-dependence into the model. In summary, the pits follow the pathway

$$
\bar{N}_{0} \stackrel{\bar{a}_{0}}{\longrightarrow} \bar{N}_{1} \stackrel{\bar{a}_{1}}{\longrightarrow} \cdots \bar{N}_{p-1} \stackrel{\bar{a}_{p-1}}{\longrightarrow} \bar{N}_{p} \stackrel{\bar{b}}{\longrightarrow}\left(\bar{L}_{i}\right) \stackrel{f \bar{k}_{i d}}{\longrightarrow} \bar{R}_{i} \stackrel{\bar{g}}{\longrightarrow} \bar{N}_{0},
$$

where $\left(\bar{L}_{i}\right)$ denotes the internalized vesicle which is a complex of internalized LDL particles and receptors, a fraction $f$ of the latter being recycled into the store $\bar{R}_{i}$. Empty internalized pits follow the simpler pathway $\bar{N}_{0} \stackrel{f \bar{b}_{0}}{\longrightarrow} \bar{R}_{i} \stackrel{\bar{g}}{\longrightarrow} \bar{N}_{0}$.

\subsection{Dynamics of LDL internalization and conversion to cholesterol}

We now construct equations for the temporal evolution of extracellular LDL $\left(\bar{L}_{e}\right)$, LDL bound to the pits $\left(\bar{L}_{b}\right)$, internalized LDL $\left(\bar{L}_{i}\right)$ and the LDL-derived intracellular cholesterol $(\bar{C})$. We recall that once the LDL particles $\left(\bar{L}_{e}\right)$ present in the extracellular medium bind to receptors in pits on the surface of hepatocytes they are internalized by the cell. Internalized LDL $\left(\bar{L}_{i}\right)$ is broken down, releasing cholesterol $(\bar{C})$ (Basu, 1984). Therefore, the LDL endocytosis can be summarized by the following set of reactions

$$
\begin{aligned}
&\text { \{extracellular medium }\} \stackrel{\bar{k}_{L}}{\longrightarrow} \bar{L}_{e} \stackrel{\left\{\bar{a}_{p}\right\}}{\longrightarrow} \bar{L}_{b} \stackrel{\bar{b}}{\longrightarrow} \bar{L}_{i} \stackrel{\bar{k}_{i d}}{\longrightarrow} \bar{r} \bar{C} \\
& \stackrel{\bar{\lambda}}{\longrightarrow}\{\text { cellular metabolism }\},
\end{aligned}
$$


where the parameters $\bar{k}_{L},\left\{\bar{a}_{p}\right\}_{p=0}^{p_{m}-1}, \bar{b}, \bar{k}_{i d}, \bar{\lambda}$ are rates, $\bar{r}$ is the number of cholesterol molecules in a typical LDL particle. Applying the law of mass action to reactions (7), we arrive at the following equations

$$
\begin{aligned}
\alpha \frac{\mathrm{d} \bar{L}_{e}}{\mathrm{~d} \bar{t}} & =\alpha \bar{k}_{L}-\bar{L}_{e} \sum_{p=0}^{p_{m}} \bar{a}_{p} \bar{N}_{p}, \\
\frac{\mathrm{d} \bar{L}_{b}}{\mathrm{~d} \bar{t}} & =\bar{L}_{e} \sum_{p=0}^{p_{m}} \bar{a}_{p} \bar{N}_{p}-\bar{b} \bar{L}_{b}, \\
\frac{\mathrm{d} \bar{L}_{i}}{\mathrm{~d} \bar{t}} & =\bar{b}_{\bar{L}_{b}}-\bar{k}_{i d} \bar{L}_{i}, \\
\frac{\mathrm{d} \bar{C}}{\mathrm{~d} \bar{t}} & =\bar{r} \bar{k}_{i d} \bar{L}_{i}-\bar{\lambda}\left(\bar{C}-\bar{C}_{e}\right),
\end{aligned}
$$

where $\alpha$ is the ratio of the volume of the extracellular medium (containing $\bar{L}_{e}$ ) and the cell volume; this takes account of the different changes in concentration which occurs as LDL crosses from the extracellular medium, is bound, and transported into the intracellular space.

The quantity $\bar{k}_{L}$ is the rate at which LDL particles are supplied to the system from an external store (e.g., replenished cell medium). Our model describes two different scenarios, depending on the value assigned to the rate $\bar{k}_{L}$. Firstly, there is the "single bolus" model, in which the hepatocytes are deprived of LDL before a fixed dose of lipoprotein is delivered at time $\bar{t}=0$ (that is, at the start of the experiment). This is simulated by fixing $\bar{k}_{L}=0$ in Eq. (8) and prescribing $\bar{L}_{e}(0)$ appropriately. The second scenario occurs when $\bar{k}_{L}>0$ so that there is a continuous source of LDL replenishing the extracellular pool of LDL. We note that $\bar{k}_{L}>0$ corresponds to the situation in which LDL particles are added continuously during the experiment. Therefore, we refer to it as the "continuouslyinfused" model. This case better reflects certain in vivo situations, in which, following a meal, lipoproteins are delivered to the liver continuously over a period of a few hours. We will investigate the single bolus and the continuously-infused models in Sections 3.1-3.2 and 3.3-4, respectively.

\subsection{Reduction of pit dynamics to a macroscopic model}

Equations (2)-(5), (8)-(11) comprise a large number of ordinary differential equations (ODEs). For example, if we assume that the maximum number of LDL particles that can be contained within one pit is $p_{m}=200$ (see Table 1), then Eqs. (2)-(5) represent 201 ODEs. In order to simplify the system, we sum Eqs. (2)-(5) and determine the evolution in time of the total number of pits occupied with any number of LDL particles bound, that is, pits are considered to be either empty, or occupied, irrespective of how much LDL is bound.

We start by assuming that the binding rate coefficient is $\bar{a}_{p}=\bar{A}\left(p_{m}-p\right)$. This is consistent with the observation that there is a maximum number of LDL particles that a pit can accommodate (Basu et al., 1978). We note that $\bar{a}_{0}=\bar{A} p_{m}$ gives the maximal rate of attachment of LDL particles. In what follows, it will be convenient to introduce two 
new variables: $\bar{M}$ representing the concentration of LDL bound in pits and $\bar{N}$ representing the total number of occupied pits. In terms of the model variables, these are defined by

$$
\bar{N}(\bar{t})=\sum_{p=1}^{p_{m}} \bar{N}_{p}(\bar{t}), \quad \text { and } \quad \bar{M}(\bar{t})=\sum_{p=1}^{p_{m}} p \bar{N}_{p}(\bar{t}) .
$$

The advantage of using these quantities is that the system of $\left(p_{m}+1\right)$ Eqs. (2)-(5), (8)(11) can be reduced exactly to just two, namely

$$
\begin{aligned}
\frac{\mathrm{d} \bar{N}_{0}}{\mathrm{~d} t} & =\bar{g} \bar{R}_{i}-\bar{A} p_{m} \bar{L}_{e} \bar{N}_{0}-\bar{b}_{0} \bar{N}_{0}, \\
\frac{\mathrm{d} \bar{N}}{\mathrm{~d} \bar{t}} & =\bar{A} p_{m} \bar{L}_{e} \bar{N}_{0}-\bar{b} \bar{N} .
\end{aligned}
$$

Simple manipulation of Eqs. (2)-(4) also results in an equation for the total number of LDL particles bound to the pits $(\bar{M})$, that is,

$$
\frac{\mathrm{d} \bar{M}}{\mathrm{~d} \bar{t}}=\bar{A} \bar{L}_{e}\left(p_{m} \bar{N}+p_{m} \bar{N}_{0}-\bar{M}\right)-\bar{b} \bar{M} .
$$

These arithmetic manipulations allow us to eliminate the microscopic quantities $\bar{N}_{p}(\bar{t})$ for $1 \leq p \leq p_{m}$ from the model. Instead of tracking the temporal evolution of the number of pits containing $p$ LDL particles for each $p$ in the range $0 \leq p \leq p_{m}$, we now track the temporal evolution of the number of pits free of $\operatorname{LDL}\left(\bar{N}_{0}\right)$, the number of pits containing LDL $(\bar{N})$, and the concentration of bound LDL $(\bar{M})$; the resulting ODEs form a closed system of equations. Therefore, Eqs. (5) and (8)-(11) become

$$
\begin{aligned}
\frac{\mathrm{d} \bar{R}_{i}}{\mathrm{~d} t} & =\frac{\bar{k}_{s}}{\bar{K}+\bar{C}}+f \bar{b} \bar{N}+f \bar{b}_{0} \bar{N}_{0}-\bar{g} \bar{R}_{i}, \\
\alpha \frac{\mathrm{d} \bar{L}_{e}}{\mathrm{~d} \bar{t}} & =\alpha \bar{k}_{L}-\bar{A} \bar{L}_{e}\left(p_{m} \bar{N}+p_{m} \bar{N}_{0}-\bar{M}\right), \\
\frac{\mathrm{d} \bar{L}_{b}}{\mathrm{~d} \bar{t}} & =\bar{A} \bar{L}_{e}\left(p_{m} \bar{N}+p_{m} \bar{N}_{0}-\bar{L}_{b}\right)-\bar{b} \bar{L}_{b}, \\
\frac{\mathrm{d} \bar{L}_{i}}{\mathrm{~d} \bar{t}} & =\bar{b} \bar{L}_{b}-\bar{k}_{i d} \bar{L}_{i}, \\
\frac{\mathrm{d} \bar{C}}{\mathrm{~d} \bar{t}} & =\bar{r} \bar{k}_{i d} \bar{L}_{i}-\bar{\lambda}\left(\bar{C}-\bar{C}_{e}\right) .
\end{aligned}
$$

We note that the concentration of LDL particles bound to the pits is consistent in Eqs. (15) and (18), that is, $\bar{L}_{b}=\bar{M}$ (henceforth, we use $\bar{L}_{b}(t)$ ).

In order to compare with Brown and Goldstein's experiments (Brown and Goldstein, 1979; Goldstein et al., 1979; Goldstein and Wofsy, 1981), we define a number of new variables that distinguish radio-labeled LDL from unlabeled LDL. In addition, we model the degradation of amino acids associated with the labeled apo B-100, assuming that this occurs at the same time and at the same rate as the release of cholesterol from LDL. 
Formally, we decompose $\bar{L}_{e}, \bar{L}_{b}$ and $\bar{L}_{i}$ into labeled and unlabeled parts, via $\bar{L}_{e}=\bar{L}_{e l}+$ $\bar{L}_{e u}, \bar{L}_{b}=\bar{L}_{b l}+\bar{L}_{b u}, \bar{L}_{i}=\bar{L}_{i l}+\bar{L}_{i u}, \bar{M}=\bar{M}_{l}+\bar{M}_{u}$, and replace (8)-(11) with

$$
\begin{aligned}
\alpha \frac{\mathrm{d} \bar{L}_{e l}}{\mathrm{~d} t} & =\alpha \bar{k}_{L l}-\bar{A} \bar{L}_{e l}\left(p_{m} \bar{N}+p_{m} \bar{N}_{0}-\bar{M}\right), & & \frac{\mathrm{d} \bar{L}_{i l}}{\mathrm{~d} \bar{t}}=\bar{b} \bar{L}_{b l}-\bar{k}_{i d} \bar{L}_{i l}, \\
\alpha \frac{\mathrm{d} \bar{L}_{e u}}{\mathrm{~d} t} & =\alpha \bar{k}_{L u}-\bar{A} \bar{L}_{e u}\left(p_{m} \bar{N}+p_{m} \bar{N}_{0}-\bar{M}\right), & & \frac{\mathrm{d} \bar{L}_{i u}}{\mathrm{~d} \bar{t}}=\bar{b} \bar{L}_{b u}-\bar{k}_{i d} \bar{L}_{i u}, \\
\frac{\mathrm{d} \bar{L}_{b l}}{\mathrm{~d} \bar{t}} & =\bar{A} \bar{L}_{e l}\left(p_{m} \bar{N}+p_{m} \bar{N}_{0}-\bar{M}\right)-\bar{b} \bar{L}_{b l}, & & \frac{\mathrm{d} \bar{A}_{i n t}}{\mathrm{~d} \bar{t}}=\gamma_{a m} \bar{k}_{i d} \bar{L}_{i l}-\bar{\lambda}^{\prime} \bar{A}_{i n t}, \\
\frac{\mathrm{d} \bar{L}_{b u}}{\mathrm{~d} \bar{t}} & =\bar{A} \bar{L}_{e u}\left(p_{m} \bar{N}+p_{m} \bar{N}_{0}-\bar{M}\right)-\bar{b} \bar{L}_{b u}, & & \frac{\mathrm{d} \bar{A}_{e x t}}{\mathrm{~d} \bar{t}}=\bar{\lambda}^{\prime} \bar{A}_{i n t}, \\
\frac{\mathrm{d} \bar{C}}{\mathrm{~d} \bar{t}} & =\bar{r} \bar{k}_{i d} \bar{L}_{i}-\bar{\lambda}\left(\bar{C}-\bar{C}_{e}\right), & &
\end{aligned}
$$

Here, $\gamma_{a m}$ is the number of labeled amino acids of apo B-100 per LDL particle, $\bar{\lambda}^{\prime}$ is the rate at which radio-labeled amino acid is transferred across the cell membrane into the extracellular medium, $\bar{k}_{L l}, \bar{k}_{L u}$ are the rates of input of labeled and unlabeled LDL into the extracellular medium.

Typical initial conditions correspond to a situation where the cells are deprived of LDL so there are no internalized LDL particles. Consequently, the pits on the cell surface are present at the maximum level $\left(\bar{n}_{0}\right)$. There is a known concentration of LDL particles in the external medium $\left(\bar{L}_{0}\right)$, all of which is unlabeled, some labeled bound LDL, but no labeled extracellular LDL, no bound unlabeled LDL, no internalized LDL of either type and because the cells have been prestarved of LDL, the intracellular cholesterol level is below its preferred level. We define $v_{p}$ to be the fraction of pits which have $p$ labeled LDL particles already bound at $t=0$. Hence, we have

$$
\bar{N}_{0}(0)=\bar{n}_{0}-\sum_{p=1}^{p_{m}} \bar{n}_{0} v_{p}, \quad \bar{N}_{p}(0)=\bar{n}_{0} v_{p} \quad\left(1 \leq p \leq p_{m}\right)
$$

which implies

$$
\begin{aligned}
& \bar{N}(0)=\sum_{p=1}^{p_{m}} \bar{n}_{0} v_{p}, \quad \bar{M}_{l}(0)=\bar{M}(0)=\bar{L}_{b 0}, \quad \bar{M}_{u}(0)=0, \\
& \bar{L}_{e l}(0)=0, \quad \bar{L}_{e u}(0)=\bar{L}_{e}(0)=\bar{L}_{0}, \quad \bar{L}_{b l}(0)=\bar{L}_{b}(0)=\bar{L}_{b 0}, \quad \bar{L}_{b u}(0)=0, \\
& \bar{L}_{i l}(0)=\bar{L}_{i u}(0)=\bar{L}_{i}(0)=0, \quad \bar{C}(0)=\theta \bar{C}_{e} \quad(0<\theta<1), \\
& \bar{R}_{i}(0)=0, \quad \bar{A}_{\text {int }}(0)=0, \quad \bar{A}_{\text {ext }}(0)=0,
\end{aligned}
$$

where $\bar{L}_{b 0}=\sum_{p=1}^{p_{m}} p \bar{n}_{0} v_{p}$.

\subsection{Parameter values}

To calculate the model parameters, we use data from the available literature where possible and for other parameters consider different parameter ranges and their impact on 
model outputs. In deriving the model, we have implicitly assumed that concentration variables are measured in number of particles per volume. Therefore, before proceeding, we need to convert all the concentrations from mass per unit volume to numbers per unit volume.

\subsubsection{Geometric parameters}

- $\bar{L}_{0}$ : Initial concentration of LDL (see Eq. (26)). In the experiments presented in Jackson et al. (2006), the initial concentration of LDL was $10 \mu \mathrm{g}$ of ApoB-100 per ml of cell medium. We convert this value to the number of particles by taking into account the molecular weight of apo B-100 particles (MW apo B-100 = 512723), that is, we write $10 \mu \mathrm{g}$ per ml of cell medium, $\bar{L}_{0}=\frac{10 \times 10^{-6}}{\mathrm{MW} \text { apo B-100 }}$ moles lipoprotein particles $/ \mathrm{ml} \times N_{A}=$ $1.17 \times 10^{13}$ particles $/ \mathrm{ml}$ of cell medium.

- $\bar{L}_{b 0}=\bar{L}_{b l}(0)$ : The initial value of bound radiolabeled LDL (see Eq. (26)); in the experiments of Brown and Goldstein (1979), this is $46 \mathrm{ng}$ of LDL ApoB per mg of cell protein (Fig. 1 of Brown and Goldstein, 1979). In the units used in this paper, this is equivalent to $1.62 \times 10^{13}$ particles per $\mathrm{ml}$ of cell volume. Note that this concentration is greater than $L_{0}$ as the reference volumes used differ.

- $\bar{n}_{0}$ : Initial number of pits (see Eq. (26)). Taking a value of $92.4 \mathrm{ng}$ of apo B-100 per mg cell protein from Harwood and Pellarin (1997), we find $\bar{R}_{T}=\frac{92.4 \times 10^{-9}}{\mathrm{MW} \text { apo B-100 }} N_{A}=$ $1.09 \times 10^{11}$ receptors per mg of cell protein, which translates to $\frac{1.09 \times 10^{11}}{\bar{V}_{c}}=3.26 \times 10^{13}$ receptors per $\mathrm{ml}$ of cell volume, using estimates of cell volumes and protein content from Table 1 . Assuming that $2 \%$ of cell area is covered by pits and using values for well protein content and well area to estimate the total number of cell receptors per well and the total number of pits per well,

$$
\begin{aligned}
R_{p i t} & =\frac{\text { total number of receptors per well }}{\text { total number of pits per well }} \\
R_{p i t} & =\frac{\bar{R}_{T} \times \text { Well Protein }}{0.02 \times \text { Well Area } / \pi r_{p i t}^{2}}=\frac{1.09 \times 10^{11} \pi(100)^{2}}{0.02}=\frac{0.2}{0.00019}=180,
\end{aligned}
$$

and, therefore, $\bar{n}_{0}=3.26 \times 10^{13} / R_{\text {pit }}=1.81 \times 10^{11}$ pits per $\mathrm{ml}$.

- $p_{m}$ : Maximum number of LDL particles in the pit; this occurs in Eqs. (13)-(15) and (17)-(18). Taking the pit radius as $100 \mathrm{~nm}$, and noting that the radius of an LDL particle is $10 \mathrm{~nm}$, we calculate that each pit can accommodate an upper limit of $2 \pi r_{p i t}^{2} / \pi r_{L D L}^{2}=$ $2\left(\frac{100}{10}\right)^{2}=200$ LDL particles.

- $\bar{V}_{c}$ : Volume of a single cell (used in calculating $\bar{V}_{\text {cell }}$, hence $\alpha$, below). We assume that one cell has approximately $300 \mathrm{pg}$ of cell protein and has a volume of $1 \mathrm{pl}$ (Table 1). Hence, $1 \mathrm{mg}$ of cell protein is equivalent to $0.0033 \mathrm{~mL}$. This conversion factor is used to convert concentrations measured relative to cell protein to that relative to cell volume.

- $\bar{V}_{c e l l}$ : The total cell culture volume is estimated from the total mass of cell protein and $\bar{V}_{c}$. Hence, for a total mass of cell protein of $0.2 \mathrm{mg}$, we estimate a cell culture volume of $0.00066 \mathrm{~mL}$. This is required for the calculation of $\alpha$, below.

- $\bar{V}_{\text {medium }}$ : We assume the volume of medium is $10 \mathrm{ml}$.

- $\alpha$ : Volume Ratio $=\bar{V}_{\text {medium }} / \bar{V}_{\text {cell }}=10 \mathrm{ml} / 0.00066 \mathrm{ml}=15000$ (nondimensional); this quantity appears in Eq. (17). 


\subsubsection{Kinetic rate parameters for $L D L$ transport across cell membrane}

- $\bar{A}$ : Rate of LDL binding to free receptors, which influences $\bar{N}_{0}, \bar{N}, \bar{M}, \bar{L}_{e}, \bar{L}_{b}$ in Eqs. (13)-(15) and (17)-(18). We equate this parameter to the rate of LDL binding to LDL-R receptors in Harwood and Pellarin (1997) (denoted by $k_{1}$ in their Table 2); and hence $\bar{A}=k_{1}=4 \times 10^{4}$ per M per sec. Since molarity, M, is the number of moles per liter, we have $\bar{A}=\frac{4 \times 10^{4}}{0.001 N_{A}}=6.64 \times 10^{-17}$ per molecule per $\mathrm{mL}$ per sec.

- $\bar{b}_{0}, \bar{b}$ : these are the internalization rates of empty, and occupied pits (and appear in all the Eqs. (13)-(19). Basu et al. (1978) took the relative lifetime of LDL bound to empty pits to be 2.27. Hence, using $\bar{b}=0.0027$ per second, from $k_{3}$ in Harwood and Pellarin (1997), and since $\bar{b}_{0}=2.27 \bar{b}$ from Basu et al. (1978), $\bar{b}_{0}=2.27 \times 0.0027=0.0061$ per second.

There is, however, a subtle difference between the definition of $k_{3}$ in Harwood and Pellarin and our parameter, $b$. Let us write the reaction scheme of Harwood and Pellarin using our notation for free, bound, internalized LDL and pits,

$$
L_{e}+R_{f} \underset{k_{2}}{\stackrel{k_{1}}{\rightleftharpoons}} L R_{b} \stackrel{k_{3}}{\rightarrow} L R_{i} \stackrel{k_{5}}{\rightarrow} L_{i}+R_{f}
$$

and compare it to ours

$$
L_{e}+R_{f} \underset{A_{-}}{\stackrel{A_{+}}{\rightleftharpoons}} L R_{b} \stackrel{b}{\rightarrow} L_{i}+R_{i} \quad \text { then } \quad R_{i} \stackrel{g}{\rightarrow} R_{f} .
$$

Note that Harwood and Pellarin have a bound complex $L_{i} R_{i}$, which we do not have, and we have recycling to an internal store, whereas they have a direct flux of receptors from the internalized complex to free receptors on the cell's surface. In our scheme, the rate of conversion of $L_{b} R_{b}$ to $L_{i}$ is simply $b$, whereas in Harwood and Pellarin (1997), it requires a combination of $k_{3}$ and $k_{5}$. The conversion of $L_{b} R_{b}$ to $R_{f}$ uses a similar combination of $k_{3}$ and $k_{5}$, but in our scheme requires a combination of $b$ and $g$. Thus, our parameters $b$ and $g$ are not directly equivalent to $k_{3}$ and $k_{5}$; they are approximations.

- $\bar{k}_{L}$ : Rate of LDL delivery, which only appears in Eq. (17) for the extracellular concentration $\bar{L}_{e}$. In order to reproduce the experimental procedures of Brown and Goldstein (1979), this rate is set equal to zero since the bolus of LDL is modeled by prescribing $\bar{L}_{e}$ at $t=0$; to approximate the in vivo situation where LDL is transported to hepatocytes over periods of several hours so that we can investigate the impact on the model results of a continuous source of lipoprotein.

\subsubsection{Kinetic parameters for the cell's internal processes}

- $\bar{g}$ : Rate of pit release from the internal store back to the cell surface, and so only influences $\bar{N}_{0}$ and $\bar{R}_{i}$, which are governed by (13) and (16), respectively. We equate this parameter to the rate of receptor recycling which has been estimated in HepG2 cells as 0.01088 per second in Harwood and Pellarin (1997).

- $\bar{C}_{e}$ : Ideal cholesterol level (see Eq. (20)). Aravindhan et al. (2006) find $56.8 \mu \mathrm{g}$ of cholesterol per mg of cell protein and taking the molecular weight of cholesterol $\left(\mathrm{MW}\right.$ chol $=386.65$, with $N_{A}$ being Avogadro's number), we obtain $\bar{C}_{e}=$ $\frac{56.8 \times 10^{-6}}{\mathrm{MW} \text { chol } \times 0.0033} N_{A}$ moles $/ \mathrm{ml}=2.65 \times 10^{19}$ molecules per $\mathrm{ml}$ of cell volume. 
Table 1 Characteristics and properties of a typical liver cell and a typical LDL particle. Values quoted are the post-optimization procedure, initial estimates for values for related systems available in the literature are quoted in brackets. Note that "mol" refers to the number of molecules or pits

\begin{tabular}{|c|c|c|}
\hline Parameter & Description & Dimensional value \\
\hline & Number of pits per cell & 180 \\
\hline & Number of receptors per cell & 32,000 \\
\hline \multirow[t]{4}{*}{$p_{m}$} & Maximum number of receptors per pit & $\sim 200$ \\
\hline & Radius of LDL particle & $10 \mathrm{~nm}$ \\
\hline & Number of receptors covered by LDL & $\sim 1$ \\
\hline & Average radius of a pit & $100 \mathrm{~nm}$ \\
\hline \multirow[t]{3}{*}{$\bar{V}_{c}$} & Average cell volume & $1 \mathrm{pl}$ \\
\hline & Average cell protein content & $300 \mathrm{pg}$ \\
\hline & Cell volume of $1 \mathrm{mg}$ cell protein & $0.0033 \mathrm{ml}$ \\
\hline $\bar{V}_{\text {medium }}$ & Volume of extracellular medium & $10 \mathrm{ml}$ \\
\hline $\bar{V}_{\text {cell }}$ & Volume of cell culture & $0.0066 \mathrm{ml}$ \\
\hline $\bar{L}_{0}$ & Initial concentration of extracellular LDL & $1.17 \times 10^{13} \mathrm{~mol} / \mathrm{ml}$ \\
\hline $\bar{n}_{0}$ & Concentration of pits & $1.81 \times 10^{11} \mathrm{~mol} / \mathrm{ml}$ \\
\hline $\bar{A}$ & Rate of LDL binding to a receptor & $6.64 \times 10^{-17} \mathrm{~mL} / \mathrm{mol} / \mathrm{sec}$ \\
\hline $\bar{g}$ & Rate of release of pits from store & 0.0108 per sec \\
\hline $\bar{b}$ & Rate of internalization of LDL-bound pits & $(0.0027) 0.0046$ per sec \\
\hline $\bar{b}_{0}$ & Rate of internalization of empty pits & 0.0061 per sec \\
\hline $\bar{k}_{L}, \bar{k}_{L l}, \bar{k}_{L u}$ & Rate of addition of LDL to medium & $0 \mathrm{~mol} / \mathrm{ml} / \mathrm{sec}$ \\
\hline $\bar{C}_{e}$ & Intracellular Cholesterol Concentration & $2.65 \times 10^{19} \mathrm{~mol} / \mathrm{ml}$ \\
\hline $\bar{K}$ & Cholesterol regulation of pit production & Varied around $\bar{C}_{e}$ \\
\hline $\bar{k}_{S}$ & Rate of production of new pits & $2.17 \times 10^{28} \mathrm{~mol}^{2} / \mathrm{ml}^{2} / \mathrm{sec}$ \\
\hline$f$ & Fraction of internalized pits recycled & {$[0.7,1]$} \\
\hline $\bar{k}_{i d}$ & Rate of degradation of LDL to cholesterol & $(0.0033) 0.0002 \mathrm{sec}^{-1}$ \\
\hline $\bar{r}$ & Number of cholesterol molecules per LDL & 3400 \\
\hline $\bar{\lambda}$ & Timescale of cholesterol regulation & 0.0033 per sec \\
\hline $\bar{\lambda}^{\prime}$ & Rate amino acid leaves the cell & $(0.0027) 0.0015$ per sec \\
\hline
\end{tabular}

- $\bar{k}_{s}$ : Maximum rate of pit production by the cell, appearing in Eq. (16). This is of the order $\mathcal{O}\left(\bar{b} \bar{n}_{0} \bar{C}_{e}\right)$ hence, $\bar{k}_{s}=2.17 \times 10^{28} \mathrm{~mol}^{2} \mathrm{ml}^{-2} \mathrm{sec}^{-1}$. In the absence of an exact value from experiments, we have optimized the fit with experimental results by varying this parameter, in conjunction with $K$ (below) by factors of up to 10 .

- $\bar{K}$ : Constant for the receptor production term in Eq. (16), this parameter should be of the order of $\mathcal{O}\left(\bar{C}_{e}\right)=10^{19}$ molecules per ml of cell volume, which we use as the base value in our simulations. For the particular form of cholesterol-regulated pit production, we use in this model and for fixed values of $\bar{k}_{s}$, larger values of $\bar{K}$ reduce the rate at which receptor production decreases with cholesterol levels. Hence, smaller values of $\bar{K}$ correspond to situations in which the cell increases pit (receptor) levels in response to deficiencies in cholesterol levels. Note that $\bar{K}$ is the cholesterol level at which the rate of de novo pit production is half its maximum.

- $f$ : Fraction of internalized receptors returned to the cell surface, which influences $\bar{R}_{i}$ through Eq. (16). Following Dunn et al. (1989), we assume $f \in[0.7,1]$.

- $\bar{k}_{i d}$ : Rate at which internalized LDL particles are degraded to release cholesterol, and hence this parameter appears in both Eqs. (19) and (20). No data is currently available, but Brown and Goldstein (1979) quote a time of 10 minutes for marked particle ingestion to measurement of related cholesterol concentration. This time includes a number 
of mechanisms included in our model and we thus estimate 5 minutes to be more realistic. Therefore, we fix $\bar{k}_{i d}=1 / 300$ per sec.

- $\bar{r}$ : Average number of cholesterol molecules per LDL particle. This only appears in the cholesterol Eq. (20). Following Jackson (2005) and Panovska et al. (2006), we take $\bar{r}=3400$.

- $\bar{\lambda}$ : Rate of removal of cholesterol from free cholesterol pool (or conversely, the fractional rate of synthesis). This parameter also only appears in Eq. (20). We assume initially that $\bar{\lambda}$ is proportional to the rate at which LDL is digested $\left(\bar{k}_{i d}\right)$; we then vary $\bar{\lambda}$ in our simulations for a best fit to the experimental data.

- $\bar{\gamma}_{a m}$ : The number of radio-labeled molecules per LDL particle, This parameter only appears in Eq. (23b) and is taken to be 1.4.

- $\vec{\lambda}$ : The rate at which amino acid products from the breakdown of cholesterol leave the cell. We vary $\bar{\lambda}^{\prime}$ in our simulations for a best fit to the experimental data. It influences both amino acid concentrations $\bar{A}_{\text {int }}$ and $\bar{A}_{\text {ext }}$ and appears in Eqs. (23b)-(24b).

The parameter values listed above are summarized in Table 1.

\subsection{Nondimensionalization of model}

We nondimensionalize Eqs. (13)-(15), (21)-(25) by introducing the following rescalings

$$
\begin{aligned}
& N_{0}=\frac{\bar{N}_{0}}{\overline{\bar{n}}_{0}}, \quad N=\frac{\bar{N}}{\overline{\bar{n}}_{0}}, \quad M=\frac{\bar{M}}{\overline{\bar{n}}_{0}}, \quad M_{l}=\frac{\bar{M}_{l}}{\bar{n}_{0}}, \quad M_{u}=\frac{\bar{M}_{u}}{\overline{\bar{n}}_{0}}, \\
& L_{e}=\frac{\bar{L}_{e}}{\bar{L}_{0}}, \quad L_{e l}=\frac{\bar{L}_{e l}}{\bar{L}_{0}}, \quad L_{e u}=\frac{\bar{L}_{e u}}{\bar{L}_{0}}, \quad C=\frac{\bar{C}}{\bar{C}_{e}}, \quad R=\frac{\bar{R}_{i}}{\bar{n}_{0}}, \\
& L_{b}=\frac{\bar{L}_{b}}{\bar{L}_{0}}, \quad L_{b l}=\frac{\bar{L}_{b l}}{\bar{L}_{0}}, \quad L_{b u}=\frac{\bar{L}_{b u}}{\bar{L}_{0}}, \quad A_{\text {int }}=\frac{\bar{A}_{i n t}}{\bar{L}_{0}}, \quad A_{\text {ext }}=\frac{\bar{A}_{e x t}}{\bar{L}_{0}} \text {, } \\
& L_{i}=\frac{\bar{L}_{i}}{\bar{L}_{0}}, \quad L_{i l}=\frac{\bar{L}_{i l}}{\bar{L}_{0}}, \quad L_{i u}=\frac{\bar{L}_{i u}}{\bar{L}_{0}}, \quad t=\bar{b} \bar{t},
\end{aligned}
$$

where overbars denote dimensional quantities and nonoverbarred quantities and parameters are used to denote their nondimensional counterparts. We choose the timescale to be that associated with pit internalization, $1 / \bar{b}$, so that the rate of internalization of nonempty pits is equal to unity in the nondimensional equations.

Rewriting Eqs. (13)-(15), (21)-(25) in terms of the dimensionless variables (29) gives the following system of dimensionless equations in which $L_{b}=\psi M$ :

$$
\begin{aligned}
\frac{\mathrm{d} N_{0}}{\mathrm{~d} t} & =g R-A p_{m} L_{e} N_{0}-b_{0} N_{0}, \\
\frac{\mathrm{d} N}{\mathrm{~d} t} & =A p_{m} L_{e} N_{0}-N, \\
\frac{\mathrm{d} M_{l}}{\mathrm{~d} t} & =A L_{e l}\left(p_{m} N+p_{m} N_{0}-M\right)-M_{l}, \\
\frac{\mathrm{d} M_{u}}{\mathrm{~d} t} & =A L_{e u}\left(p_{m} N+p_{m} N_{0}-M\right)-M_{u}, \\
\frac{\mathrm{d} R}{\mathrm{~d} t} & =\frac{k_{r}}{K+C}+f N+f b_{0} N_{0}-g R,
\end{aligned}
$$


Table 2 Nondimensional parameters for the model of LDL endocytosis (note that all rates are relative to the rate of internalization of occupied pits)

\begin{tabular}{lll}
\hline Parameter & Description & Value \\
\hline$p_{m}$ & Number of receptors in a pit & 200 \\
$\alpha$ & Volume ratio of extracellular to cellular media & 15,000 \\
$g$ & Rate of return of internal pits to surface & 2.35 \\
$A$ & Rate of LDL binding & 0.17 \\
$b_{0}$ & Rate of internalization of empty pits & 1.32 \\
$k_{L} k_{L u}, k_{L l}$ & Rate of delivery of LDL to extracellular medium & 0 \\
$K$ & Determines cholesterol dependence of pit production & 2.3 \\
$k_{r}$ & Rate of production of new pits & 0.235 \\
$f$ & Fraction of internalized pits which are recycled & 0.7 \\
$k_{i d}$ & Rate of conversion of LDL to cholesterol & 0.0435 \\
$r$ & Cholesterol content per LDL particle & 0.0015 \\
$\lambda$ & Rate of cholesterol regulation & 0.717 \\
$\lambda^{\prime}$ & Rate at which labeled amino acids leave cell & 0.326 \\
$\psi$ & Ratio of pits to LDL particles at $t=0$ & 0.0155 \\
$\gamma_{a m}$ & Number of tagged amino acids per LDL & 1.4 \\
$\theta$ & Initial internal cholesterol level as a fraction of equilibrium & 0.7 \\
$\delta$ & Ratio of radiolabeled: unlabeled LDL & 1.38 \\
\hline
\end{tabular}

$$
\begin{aligned}
\alpha \frac{\mathrm{d} L_{e l}}{\mathrm{~d} t} & =\alpha k_{L l}-A \psi L_{e l}\left(p_{m} N+p_{m} N_{0}-M\right), \\
\alpha \frac{\mathrm{d} L_{e u}}{\mathrm{~d} t} & =\alpha k_{L u}-A \psi L_{e u}\left(p_{m} N+p_{m} N_{0}-M\right), \\
\frac{\mathrm{d} L_{i l}}{\mathrm{~d} t} & =\psi M_{l}-k_{i d} L_{i l}, \\
\frac{\mathrm{d} L_{i u}}{\mathrm{~d} t} & =\psi M_{u}-k_{i d} L_{i u}, \\
\frac{\mathrm{d} C}{\mathrm{~d} t} & =r k_{i d} L_{i}-\lambda(C-1), \\
\frac{\mathrm{d} A_{i n t}}{\mathrm{~d} t} & =\gamma_{a m} k_{i d} L_{i l}-\lambda^{\prime} A_{i n t}, \\
\frac{\mathrm{d} A_{e x t}}{\mathrm{~d} t} & =\lambda^{\prime} A_{i n t},
\end{aligned}
$$

together with the initial conditions

$$
\left.\begin{array}{l}
N_{0}(0)=1-\sum_{p=1}^{p_{m}} v_{p}, \quad N(0)=\sum_{p=1}^{p_{m}} v_{p}, \quad M(0)=M_{l}(0)=\frac{\delta}{\psi}, \\
M_{u}(0)=0, \quad R^{2}, \quad(0)=0, \\
L_{e}(0)=1, \quad L_{e l}(0)=0, \quad L_{e u}(0)=1, \\
L_{b}(0)=L_{b l}(0)=\delta, \quad L_{b u}(0)=0, \\
L_{i}(0)=L_{i l}(0)=L_{i u}(0)=0, \quad A_{\text {int }}(0)=0, \quad A_{\text {ext }}(0)=0, \quad C(0)=\theta ;
\end{array}\right\}
$$


since $L_{b}=\psi M$, the initial conditions (26) imply $\delta=\psi \sum_{p=1}^{p_{m}} p v_{p}$. The new nondimensional parameters appearing above are defined by

$$
\begin{array}{lrrr}
A=\frac{\bar{A} \bar{L}_{0}}{\bar{b}}, & \psi=\frac{\bar{n}_{0}}{\bar{L}_{0}}, & b_{0}=\frac{\bar{b}_{0}}{\bar{b}}, \quad g=\frac{\bar{g}}{\bar{b}}, \quad k_{r}=\frac{\bar{k}_{s}}{\bar{n}_{0} \bar{b} \bar{C}_{e}}, \\
K=\frac{\bar{k}_{L}}{\overline{\bar{C}}_{e}}, & k_{L}=\frac{\bar{k}_{L l}}{\overline{\bar{L}}_{0} \bar{b}}, & k_{L l}=\frac{\bar{k}_{L u}}{\bar{L}_{0} \bar{b}}, & k_{L u}=\frac{\overline{k_{L u}}}{\bar{L}_{0} \bar{b}}, \\
k_{i d}=\frac{\bar{k}_{i d}}{\bar{b}}, & r=\frac{\bar{\lambda}}{\bar{C}_{e}}, & \lambda=\frac{\overline{\bar{\lambda}}}{\bar{b}}, \quad \lambda^{\prime}=\frac{\bar{L}_{b 0}}{\bar{b}}, \quad \delta=\frac{\bar{L}_{0}}{}
\end{array}
$$

The calculated nondimensional values used in the numerical simulations are listed in Table 2.

\section{Numerical results}

In this section, we present numerical solutions of Eqs. (30)-(41). Since the system of equations cannot be solved analytically, the numerical solver ode45 (in Matlab) is used to study the evolution in time of the model variables. In the first instance, using the parameters in Tables 1 and 2 as a starting point, we focus on Brown and Goldstein's classical experiments (Brown and Goldstein, 1979). In order to reproduce their results (and to calibrate our model), we need to vary some of the system parameters. We use fminsearch in Matlab to minimize the $\mathbb{R}^{2}$ difference between experimental and simulation values. In Brown and Goldstein (1979), the dynamics of binding, internalization, and degradation of LDL by LDL-R mediated uptake were studied using radio labeled LDL ( $\left.{ }^{125} \mathrm{I}-\mathrm{LDL}\right)$. Fibroblasts were incubated at $4{ }^{\circ} \mathrm{C}$ with ${ }^{125} \mathrm{I}-\mathrm{LDL}$ so that binding, but not internalization occurred. This resulted in labeled ${ }^{125} \mathrm{I}$-LDL particles being bound to the cell surface with concentration $46 \mathrm{ng} / \mathrm{mg}$ cell protein. The cells were then washed before being incubated in a medium containing $10 \mu \mathrm{g}$ of unlabeled LDL per ml. The cells were then warmed to $37^{\circ} \mathrm{C}$ to allow internalization and degradation of LDL to commence. At a number of time points over a 2-hour period, the concentrations of surface-bound, internalized, and culture medium ${ }^{125}$ I were measured. The appearance of label in the medium was due to the degradation of amino acids of apo B-100 within lysosomes in the cells (which we assume occurs at the same time that cholesterol esters are hydrolyzed) and their subsequent export across the cell membrane into the culture medium.

The dynamics of the model as illustrated in Fig. 2 fit reasonably well with data from Brown and Goldstein (1979) for bound and internalized ${ }^{125}$ I-LDL, and external amino acid behavior. The model, however, shows a persistent mismatch between the long time concentration of bound labeled ${ }^{125} \mathrm{I}-\mathrm{LDL}$ which plateaus at a nonzero value and that predicted by the theory (which decays to zero within the first 1,000 second). The reason for this mismatch could be either a systematic error in the experiment or some additional process not incorporated in the model. Our model is based on the assumption that all bound ${ }^{125} \mathrm{I}-\mathrm{LDL}$ particles are located within pits on the surface of hepatocytes, but it is possible that ${ }^{125} \mathrm{I}-\mathrm{LDL}$ also attaches in a nonspecific manner to other parts of the cell membrane. Our model does not allow for such effects even though it has been reported previously (see, for example, Galeano et al., 1998, particularly Fig. 2 therein). This type of LDL binding does not result in cellular uptake, and so causes the surface bound ${ }^{125}$ I-LDL to remain elevated during the later stages of the experiment $(2,000<t<7,200$ seconds $)$. 


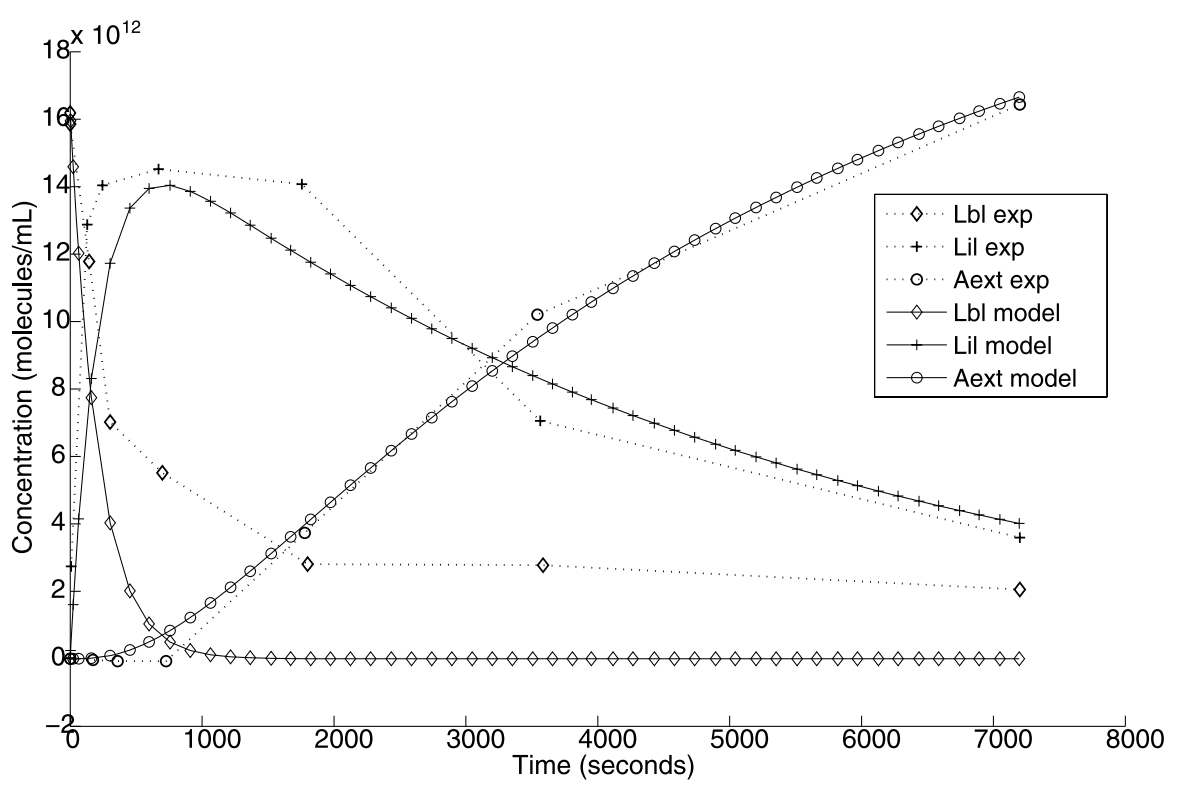

Fig. 2 Comparison of experimental data with the optimized model output using final dimensional parameter values in Table 1. The dotted line with diamonds corresponds to the experimentally measured concentration of bound labeled LDL; the dotted line marked with ' + ' denotes the experimentally measured concentration of labeled internalized LDL, and the dotted line with open circles denotes the concentration of labeled amino acids in the extracellular medium. The solid lines with these symbols correspond to the same quantities in our numerical simulation of the model equations.

\subsection{Evolution of the system}

In our simulations (Fig. 3), we assume that the system reaches a steady state within the experimental timeframe of 2 hours. In order to achieve this, we set the fraction of internalized pits recycled to the store to $f=0.7$ (with larger values of $f$ our simulations show that the total number of pits $N_{0}+N+R_{i}$ does not reach a steady-state over the 2-hour time-course of the experiment).

There is a reduction in the total number of receptors (and pits) as cholesterol enters the cell within LDL, due to the imperfect recycling of receptors (pits); this is counterbalanced by the cholesterol-regulated production term $k_{s} /(K+C)$. The parameters $k_{s}$ and $K$ are chosen so that the total number of receptors (and thus pits) are maximal initially, to reflect the initial conditions of the experiment in which cells are incubated in a lipid-poor medium to upregulate LDL receptor levels. (See Section 4.2 for more details.)

From the top left graph of Fig. 3, we see that at small times, as extracellular LDL $\left(L_{e}\right)$ binds to receptors the number of empty pits drops rapidly and the number of occupied pits increases. $N$ is observed to overshoot its steady-state value, as internalization occurs on a slightly longer timescale than adhesion. The system then enters a quasi-steady state in which the number of internalized pits $\left(R_{i}\right)$ is about $0.5 \times 10^{11}, N \approx 1.2 \times 10^{11}$ and $N_{0}<0.1 \times 10^{11}$. During this steady phase, the production of new pits $\left(\bar{k}_{s} /(\bar{K}+\bar{C})\right)$ balances the loss due to imperfect recycling of internalized pits, $(1-f)\left(\bar{b} \bar{N}+\bar{b}_{0} \bar{N}_{0}\right)$. 

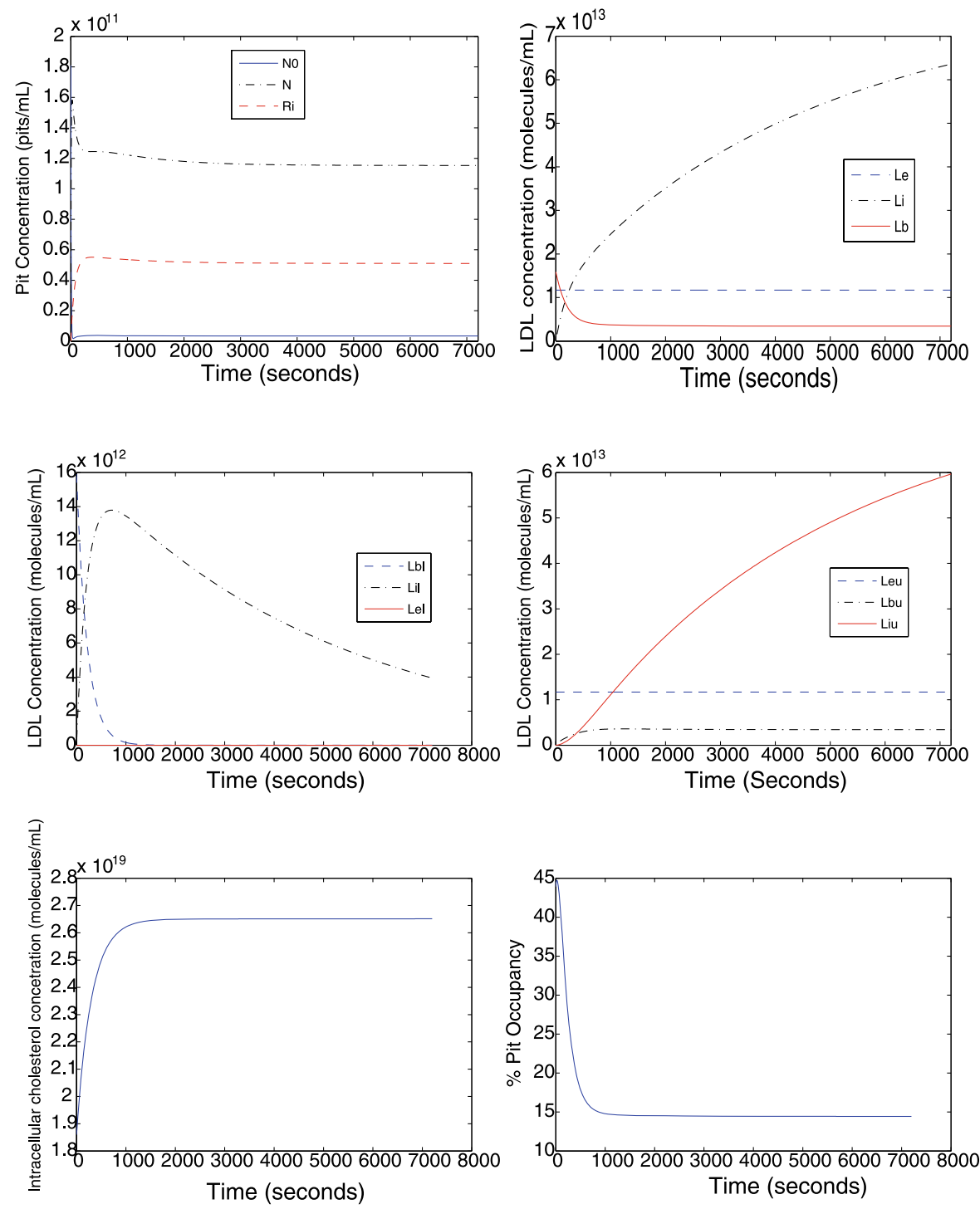

Fig. 3 Pit, LDL and cholesterol concentrations using final parameter values in Table 1. The value of $K$ is set equal to $2.94 \times 10^{18}$ and the value of $k_{S}$ to $4.87 \times 10^{27}$ so that pit production is equal to $10 \%$ of its maximum value when $C=C e$ and the final steady state concentration of pits $\left(N_{0}+N+R_{i}\right)$ is $95 \%$ of its initial value. Top left: concentrations of occupied,internalized, and empty pits $\left(\bar{N}, \bar{R}_{i}, \bar{N}_{0}\right)$ plotted against time; Top right: concentrations of internalized, extracellular, and bound LDL $\left(\bar{L}_{i}, \bar{L}_{e}, \bar{L}_{b}\right)$ against time; Center left: concentrations of labeled internalized and bound LDL $\left(\bar{L}_{i l}, \bar{L}_{b l}\right)$ against time (the trace for the concentration of labeled extracellular LDL $\bar{L}_{e l}$ is zero throughout); Center right: concentrations of unlabeled internalized, extracellular and bound LDL $\left(\bar{L}_{i u}, \bar{L}_{e u}, \bar{L}_{b u}\right)$ against time; Bottom left: plot of intracellular cholesterol level $(\bar{C})$ against time, Bottom right: plot of pit occupancy percentage $\left(100 \bar{M} / p_{m}\left(\bar{N}+\bar{N}_{0}\right)\right)$ against time. 
Total extracellular LDL $\left(L_{e}\right)$ remains almost constant showing very little depletion from such a large external source. Labeled bound LDL falls from its initial concentration, at the same rate as it is internalized, ultimately this falls to zero since no more labeled LDL is introduced into the medium. Unlabeled bound LDL initially increases due to a high percentage of pits being recycled back to the surface, this allows further binding of unlabeled LDL until the maximum number of LDL particles per pit is reached and the faster rate of internalization takes over.

Overall, total bound LDL drops from our initial measure of labeled bound LDL to a steady state value of unlabeled bound LDL produced by the model. Labeled bound LDL gets taken up, producing labeled internalized LDL. When all the labeled bound LDL is internalized, the concentration $L_{i l}$ decreases at a rate set by the degradation of LDL and the internalization of new unlabeled LDL. The total internalized LDL does not reach equilibrium until much later (6-12 hours) which is surprising considering cholesterol reaches a quasi-steady state around half an hour in to the simulation. One might assume that cholesterol levels depend on the internalization rate of LDL and the behavior of $L_{i}$. However, our model shows this is not the case. Sensitivity analysis in Section 3.2 shows that the intracellular cholesterol level is predominantly determined by its rate of regulation $(\lambda)$ and its initial concentration whereas $L_{i}$ is (indirectly) dependent on many rate parameters, which cause a long transient state (pit production, max LDL per pit, binding, recycling pits and fraction of those returned, as well as degradation and internalization of LDL). This effect can be seen in Fig. 3: compare $\bar{L}_{i}$ in the top right graph with $\bar{C}$ in the bottom left graph. The cholesterol concentration evolves over two timescales, it rapidly approaches a quasi-steady-value given by

$$
C=1+\frac{r k_{i d} L_{i}(t)}{\lambda}
$$

due to cholesterol synthesis, or removal of cholesterol from intracellular stores, and not the internalization of LDL. Later, as $L_{i}(t)$ changes over a much slower timescale, $C$ also changes.

\subsection{Sensitivity analysis}

Sensitivity analysis examines how, near the chosen set of parameter values, a small variation in each model parameter affects the model outputs. For example, how the labeled internalized LDL concentration $\left(\bar{L}_{i l}\right)$ varies with the rate of conversion of internal LDL to cholesterol $\left(k_{i d}\right)$; we denote such a quantity by $S\left(\bar{L}_{i l}, \bar{k}_{i d}, t\right)$ since it is a time-dependent quantity.

The time-dependent normalized (and hence dimensionless) sensitivity coefficient $S(x, p, t)$ is defined by

$$
S(x, p, t)=\frac{\mathrm{d} \log x(t ; p)}{\mathrm{d}(\log p)}=\frac{p}{x(t ; p)} \frac{\mathrm{d} x(t ; p)}{\mathrm{d} p},
$$

where $x$ is a model variable and $p$ denotes a parameter. Hence, a value of zero indicates that the variable $x(t ; p)$ is insensitive to changes in the value of $p$. Conversely, a value of unity indicates that a change in the value of $p$ causes an equivalent proportional change in the model variable. If the absolute value of the sensitivity coefficients is greater than 
Table 3 Table of the measured variables (observed concentrations) and the parameters which they are sensitive to; parameters are listed in order of decreasing sensitivities

\begin{tabular}{ll}
\hline Variable & Parameters exerting the dominant influence \\
\hline $\bar{L}_{b l}$ & $\bar{b}$ \\
$\bar{L}_{i l}$ & $\bar{b}, \bar{k}_{i d}$ \\
$\bar{A}_{e x t}$ & $\bar{b}, \bar{k}_{i d}, \bar{\gamma}_{a m}, \bar{\lambda}^{\prime}$ \\
\hline
\end{tabular}
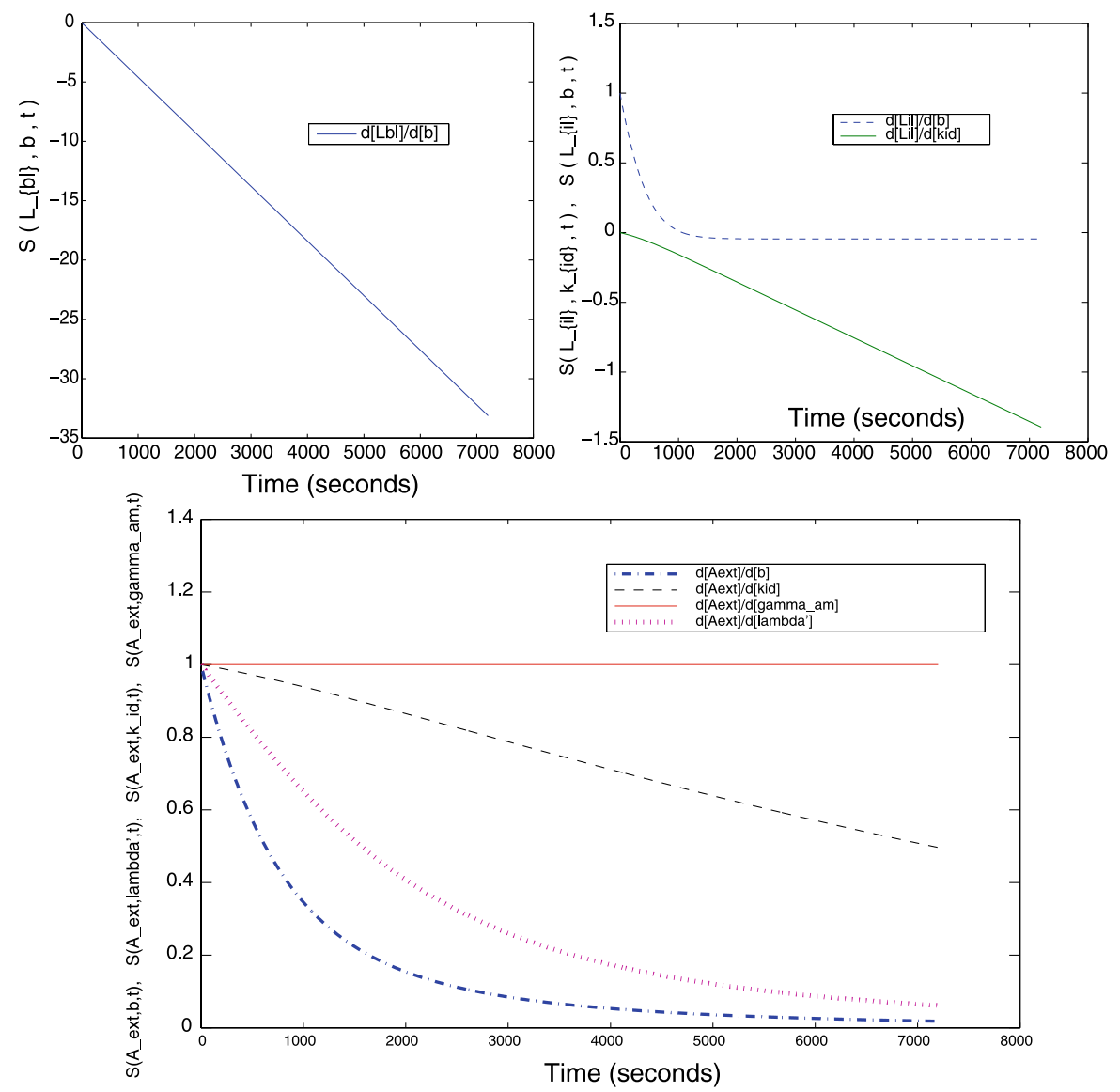

Fig. 4 Sensitivity plots. Top left: The sensitivity $S\left(\bar{L}_{b l}, \bar{b}, t\right)$ of the concentration of bound labeled LDL $\left(\bar{L}_{b l}\right)$ with the internalization rate parameter $(\bar{b})$ plotted as a function of time, from the definition (45); Top right: the sensitivities $S\left(\bar{L}_{i l}, \bar{b}, t\right)$ and $S\left(\bar{L}_{i l}, \bar{k}_{i d}, t\right)$ of the labeled internalized LDL concentration $\left(\bar{L}_{i l}\right)$ with the internalization rate $(\bar{b})$ and the LDL degradation rate $\left(\bar{k}_{i d}\right)$ as a function of time; Bottom: sensitivities of the labeled extracellular amino acid concentration $\left(\bar{A}_{\text {ext }}\right)$ with the internalization rate $(\bar{b})$, the LDL degradation rate $\left(\bar{k}_{i d}\right)$, the number of tagged amino acid molecules per LDL particle $\left(\gamma_{a m}\right)$, and the amino acid transport rate $\left(\bar{\lambda}^{\prime}\right)$, all as functions of time $(\bar{t})$. 
Table 4 Table of the key parameters identified by sensitivity analysis which influence variables in the model. Determination of the "most influential" is based on the absolute maximum of $S(t)$ over the 2-hour period

\begin{tabular}{ll}
\hline Variable & Parameters which influence variable \\
\hline Pit concentrations, $\bar{N}, \bar{N}_{0}, \bar{R}$ & $f, \bar{g}, \bar{A}, p_{m}, \bar{b}_{0}, \bar{b}, \bar{k}_{s}, \bar{K}, \bar{C}_{e}$, \\
LDL concentrations $\bar{L}_{b}, \bar{L}_{i}$ & $(f$ is the most influential $)$ \\
& $f, \bar{g}, \bar{A}, p_{m}, \bar{b}_{0}, \bar{b}, \bar{k}_{s}, \bar{K}_{i d}, \bar{C}_{e}$, \\
Cholesterol $\bar{C}$ & $(f$ is the most influential, followed by $\bar{b})$ \\
Amino acid concentration, $\bar{A}_{i n t}+\bar{A}_{e x t}$ & $\bar{\lambda}, \bar{C}_{e},\left(\bar{C}_{e}\right.$ being the more influential $)$ \\
\hline
\end{tabular}

unity, this indicates that changes in $p$ cause disproportionately larger changes in the model output. Negative values of the sensitivity imply that an increase in the parameter causes a decrease in the variable.

Since sensitivity analysis involves a calculation of how each initial condition and each parameter used in the model influences each model variable at each moment in time, vast amounts of data are generated. Much of this reinforces the fact that many of the processes are not rate-limiting, and so changing one rate or initial condition has little or no effect on the model variable. Here, we summarize those dependencies which sensitivity analysis has shown to be the most significant.

In Brown and Goldstein (1979), three quantities are measured: the concentration of surface bound labeled LDL $\left(\bar{L}_{b l}\right)$, internalized labeled LDL $\left(\bar{L}_{i l}\right)$ and amino acid degradation products of the radio-labeled apo B-100 in the external medium $\left(\bar{A}_{\text {ext }}\right)$.

The results of sensitivity analysis are shown in Fig. 4 for the three model variables associated with the observables in the experimental data and using the parameters in Table 1. For $L_{b l}, L_{i l}$ and $A_{e x t}$, we find that these model outputs are only sensitive to variations in four parameters (see Table 3 and Fig. 4 for details). All other parameters have sensitivity coefficients near zero and, therefore, do not have any effect on the concentration variables.

Hence, we find that the rate of internalization $(\bar{b})$ exclusively determines how the concentration of labeled bound LDL changes with time, but also has a significant influence on the other two measured variables, the labeled internalized LDL concentration $\left(\bar{L}_{i l}\right)$ and the extracellular concentration of labeled amino acids $\left(\bar{A}_{\text {ext }}\right)$. The rate at which LDL is degraded $\left(\bar{k}_{i d}\right)$ significantly influences both the concentration of internalized LDL and degradation products in the external medium $\left(\bar{L}_{i l}\right.$ and $\bar{A}_{\text {ext }}$, respectively). Sensitivity analysis of the remaining model variables to changes in the other parameters highlights the next most influential parameters. Of these, the most important is the fraction of pits returned to the cell surface $(f)$, or inversely, the fraction degraded/lost from the cell. For both, the number of pits (internal, empty, and occupied) and the number of LDL particles (bound and internalized) an increase in this fraction causes a two-fold increase in their concentration from half an hour onward. Sensitivity analysis aids the process of optimizing parameter values to reproduce experimental data since it highlights those parameters which have the greatest influence on each component of the system.

From a total of 18 model parameters, 9 are commonly identified as parameters crucial to the uptake of LDL (see Table 4, which lists variables in our model for which there is no experimental data). Pit and LDL concentrations are determined by the same 9 parameters except for the rate of LDL degradation to cholesterol $\left(k_{i d}\right)$ which not surprisingly, decreases the concentration of internalized LDL. The rate of internalization $(\bar{b})$ is identified 
as the next most important parameter influencing bound, internalized LDL and pit concentrations. The remaining parameters have sensitivities of order one. The rate of binding $(\bar{A})$ and maximum number of LDL allowed per pit $\left(p_{m}\right)$ have little effect on pits other than at very early times where they cause a dramatic increase in bound pits. This is because it is only at early times that $N_{0}$ is significantly different from zero. Cholesterol is surprisingly only dependent on its rates of synthesis and utilization, which we model as a single regulation term, and its initial concentration, and not the rate of LDL-degradation as might be expected.

\subsection{Kinetics of convergence to quasi-steady-states}

Having identified the recycling fidelity parameter $f$ as the dominant parameter in the fitting of experimental data, we now investigate its role in determining the rate at which the system attains its steady-state.

A low value of $f$ means that there is a high rate of degradation of pits, and consequently a high rate of pit production is required to maintain a steady-state for the total number of pits. In this case, the system rapidly equilibrates, (see the lower panel of Fig. 5), a behavior we describe as responsive or flexible. Correspondingly, a low degradation rate (meaning $f \approx 1$ ) requires only a low pit production rate to sustain the steady-state. This gives rise to a system which takes longer to converge to steady-state (see the lower panel of Fig. 5). Such systems may be described as inflexible. However, since receptors are recycled with a high degree of fidelity, few new receptors are required, and little energy is expended making them. In contrast, when $f$ is low, the cell must bear the cost of manufacturing many more receptors de novo.

The upper panel of Fig. 5 shows the differences in the concentrations of internalized LDL in the two cases. The low degradation case $(f \approx 1)$ leads to significantly higher values of $L_{i}$ than the high degradation case. This suggests that the inflexible case is the more efficient in its uptake of LDL. Cells balance the need for efficient uptake of LDL, the cost of de novo manufacture of receptors, and their ability to respond to an environment of variable LDL concentration. There is a balance to be struck between timescale of equilibration on one hand and on the other, the speed of LDL uptake and cost of receptorsynthesis.

\section{Steady-state behavior}

Since there are no published experimental results for the case where LDL is continuously supplied to the extracellular medium, we no longer consider the concentrations of radiolabeled species. Instead of the full system of Eqs. (30)-(41), in this section we focus on the simpler system

$$
\begin{aligned}
\frac{\mathrm{d} N_{0}}{\mathrm{~d} t} & =g R-A p_{m} L_{e} N_{0}-b_{0} N_{0}, \\
\frac{\mathrm{d} N}{\mathrm{~d} t} & =A p_{m} L_{e} N_{0}-N, \\
\frac{\mathrm{d} M}{\mathrm{~d} t} & =A L_{e}\left(p_{m} N+p_{m} N_{0}-M\right)-M,
\end{aligned}
$$



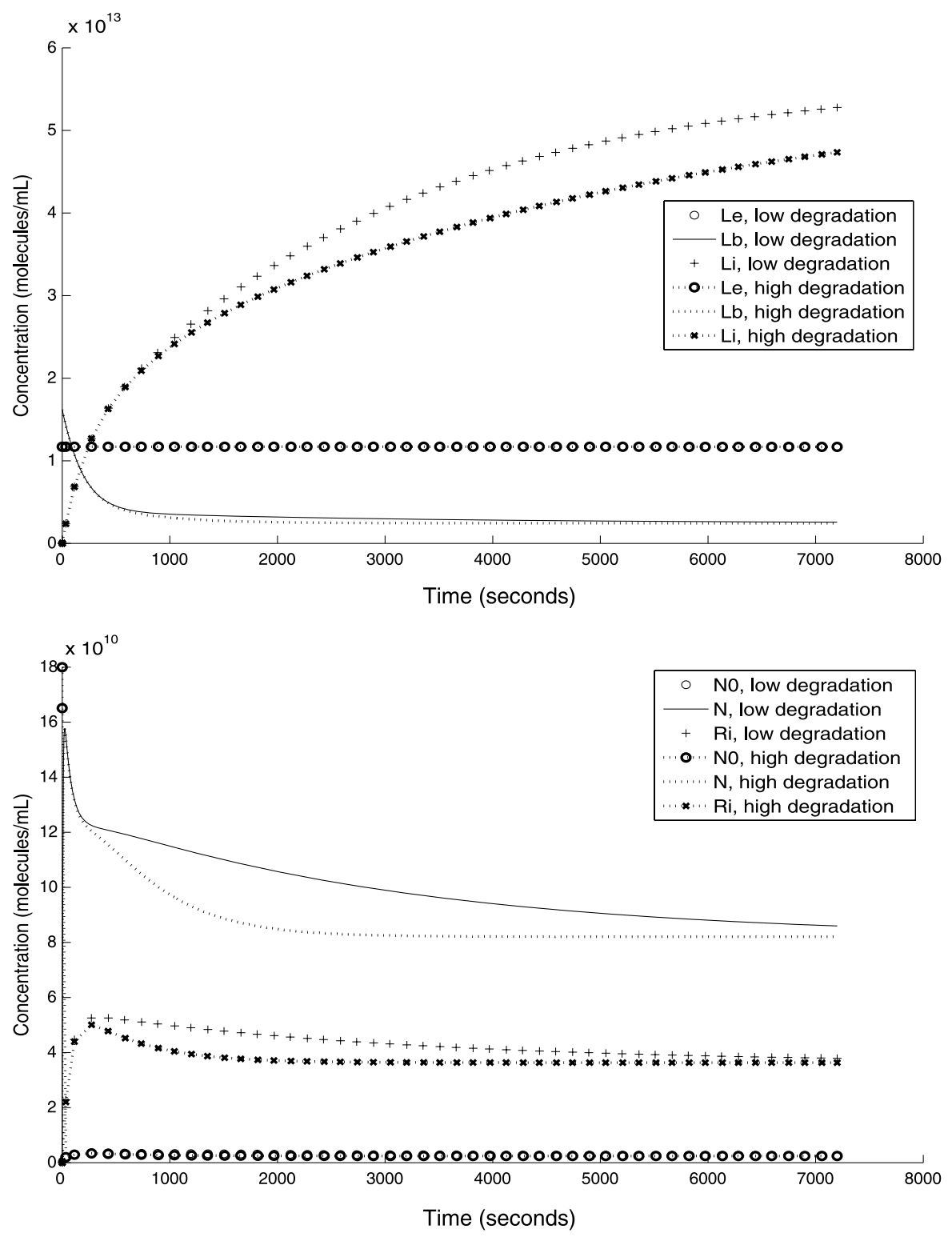

Fig. 5 Plots indicating the effect of the recycling fidelity parameter $f$ on the kinetics of convergence to steady-state. Top: graphs of $L_{e}, L_{b}$, and $L_{i}$ against time, $t$. Bottom: graphs of $N_{0}, N$, and $R_{i}$ against time, $t$. In both cases, the curve rapidly approaching steady-state corresponds to $f=0.1$, and that showing a slow convergence to steady-state is for $f=0.9$. The pit-production rate $k_{r}$ is adjusted so that in both cases, the steady-state total number of pits is $70 \%$ of the initial number (that is, $k_{s}=1.08 \times 10^{28} \mathrm{~mol}^{2} / \mathrm{ml}^{2} / \mathrm{sec}$ and $k_{s}=1.19 \times 10^{27} \mathrm{~mol}^{2} / \mathrm{ml}^{2} / \mathrm{sec}$, where $K=2.94 \times 10^{18} \mathrm{~mol} / \mathrm{ml}$; see Section 4.2 and, in particular, Eq. (75) for more details. 


$$
\begin{aligned}
\frac{\mathrm{d} R}{\mathrm{~d} t} & =\frac{k_{r}}{K+C}+f N+f b_{0} N_{0}-g R, \\
\alpha \frac{\mathrm{d} L_{e}}{\mathrm{~d} t} & =\alpha k_{L}-A \psi L_{e}\left(p_{m} N+p_{m} N_{0}-M\right), \\
\frac{\mathrm{d} L_{i}}{\mathrm{~d} t} & =\psi M-k_{i d} L_{i}, \\
\frac{\mathrm{d} C}{\mathrm{~d} t} & =r k_{i d} L_{i}-\lambda(C-1),
\end{aligned}
$$

still with initial conditions given by (42).

\subsection{Fixed number of pits $\left(f=1, k_{r}=0\right)$}

Here, we show that our model can reproduce the results of Harwood and Pellarin's (1997) model. Harwood and Pellarin quote values for the proportion of receptors which are free, bound, and internalized as a function of the extracellular concentration, assuming the system has reached steady-state. They considered a system in which the total number of pits or receptors was in steady-state, and hence constant. We reproduce this behavior by assuming perfect recycling of pits $(f=1)$ and neglecting the de novo synthesis of pits (that is, we impose $k_{r}=0$ ). In Harwood and Pellarin (1997) the internalization of empty pits was neglected and so we fix $b_{0}=0$.

Harwood and Pellarin (1997) assumed a steady-state model in which $L_{e}$ is assumed to be constant, and hence we ignore Eq. (50). This can be derived from (50) by assuming that there is no input of LDL, hence we set $k_{L}=0$; furthermore, since $\alpha \gg 1$, the uptake of LDL by cells has minimal effect on the concentration $L_{e}$. The leading-order approximation of (50) is thus $\mathrm{d} L_{e} / \mathrm{d} t=0$, giving $L_{e}$ constant. We assume the system will asymptote to a steady-state, and so instead of (46)-(52), we consider the further reduction to

$$
\begin{aligned}
& 0=g R-A p_{m} L_{e} N_{0}, \\
& 0=A p_{m} L_{e} N_{0}-N, \\
& 0=A L_{e}\left(p_{m} N+p_{m} N_{0}-M\right)-M, \\
& 0=N-g R, \\
& 0=\psi M-k_{i d} L_{i}, \\
& 0=r k_{i d} L_{i}-\lambda(C-1) .
\end{aligned}
$$

Note that this system of six equations are not independent, (56) is redundant, being the sum of (53) and (54). In the case $k_{r}=0, f=1$, the total number of receptors is conserved by (46)-(52), and so determined by the initial data imposed, not by the Eqs. (53)-(58).

Since $\alpha \gg 1$, we treat $L_{e}$ as a prescribed parameter, and calculate the proportion of free bound and internalized receptors as 


$$
\begin{aligned}
& \Phi_{\text {free }}=\frac{p_{m} N+p_{m} N_{0}-M}{p_{m}\left(R+N+N_{0}\right)}, \quad \Phi_{\text {bound }}=\frac{M}{p_{m}\left(R+N+N_{0}\right)}, \\
& \Phi_{\text {int }}=\frac{R}{R+N+N_{0}} .
\end{aligned}
$$

Solving the steady-state Eqs. (53)-(58), we find

$$
\begin{aligned}
\Phi_{\text {free }} & =\frac{g\left(1+A p_{m} L_{e}\right)}{\left(1+A L_{e}\right)\left[g+(1+g) A p_{m} L_{e}\right]}, \\
\Phi_{\text {bound }} & =\frac{A g L_{e}\left(1+A p_{m} L_{e}\right)}{\left(1+A L_{e}\right)\left[g+(1+g) A p_{m} L_{e}\right]}, \\
\Phi_{\text {int }} & =\frac{A p_{m} L_{e}}{g+(1+g) A p_{m} L_{e}} .
\end{aligned}
$$

In Fig. 6, these quantities are plotted against a wide range of values of $L_{e}$ to show that our model agrees with that of Harwood and Pellarin (1997). The value of $L_{e}$ most commonly used in this paper corresponds to an external LDL concentration of $50 \mu \mathrm{g} / \mathrm{ml}$ which, according to Harwood and Pellarin's figures, gives 30\% bound, 10\% internalized and $60 \%$ free receptors, this is at the smaller end of $L_{e}$ values displayed in Fig. 6. Since the rate of pit-internalization $(\bar{b})$ used in this paper differs that of Harwood and Pellarin (1997), our figures differ slightly from theirs. As the external LDL concentration increases, we observe a monotonic decrease in free receptors to below 10\%, as do Harwood and Pellarin. Similarly, both models predict a sharp increase in the fraction of receptors with bound LDL from zero to over $70 \%$. Both models also predict that during the steady-state, approximately $20 \%$ of all receptors are internalized, and that this proportion does not vary with the concentration of extracellular LDL. The remaining $80 \%$ of receptors are to be found on the surface, and the proportions which are empty or occupied is dependent on the extracellular concentration of LDL.

In addition, since our model takes account of the number of receptors in each pit, we can calculate the average occupancy of a pit. This is defined by $M /\left(N+N_{0}\right)$; as a percentage of the maximum occupancy $\left(p_{m}\right)$, we plot

$$
\% \text { age Occupancy }=\frac{100 M}{p_{m}\left(N+N_{0}\right)}=\frac{100 A L_{e}}{1+A L_{e}},
$$

in Fig. 6. This shows that the occupancy rises with external LDL concentration, but extremely large concentrations are required to achieve $50 \%$ of receptors bound. We can only speculate on the reasons for such a surprising low occupancy: it may be related to the historical need for flexibility in the uptake of LDL, that is, occasionally the system had to deal with large concentrations of LDL, or that the LDL-receptor is also used for the uptake of other particles. In Pearson et al. (2008) and Tindall et al. (2008), we analyze generalized models in which VLDL particles preferentially bind to LDL-receptors causing LDL to compete for receptors.

\subsection{Variable number of pits $\left(f<1, k_{s}>0\right)$}

In the above case, the number of receptors was fixed and determined by the initial conditions. Our more general model allows for a variable number of receptors since it includes 


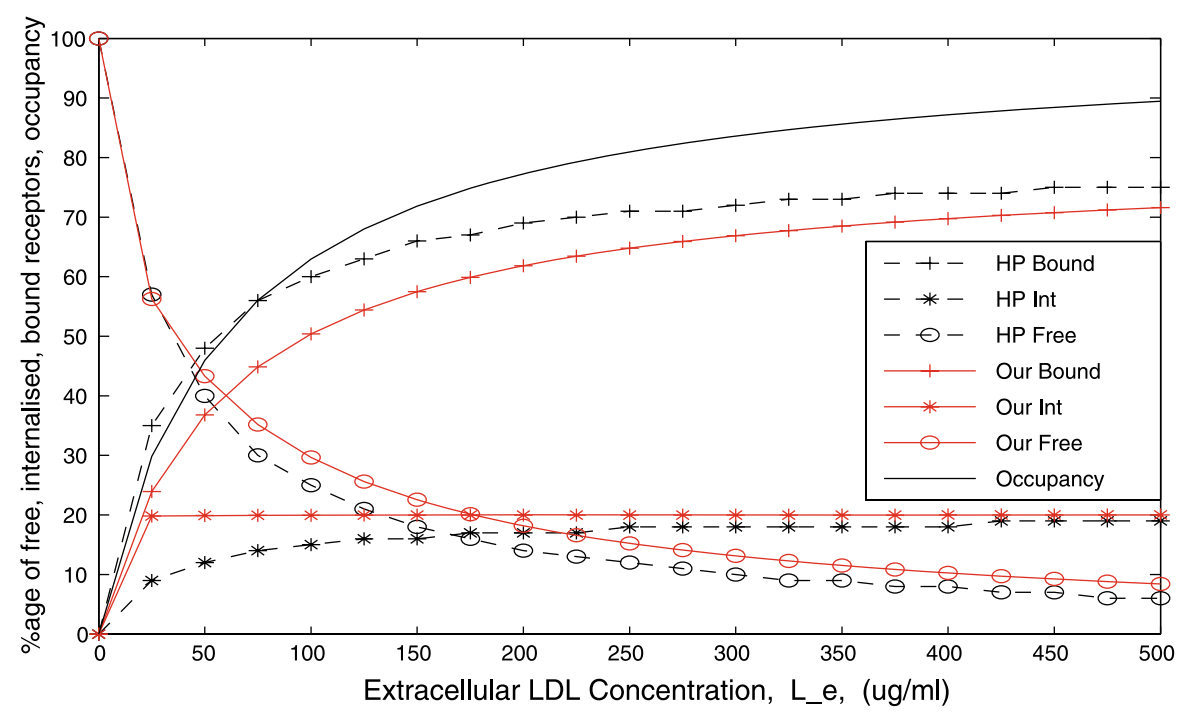

Fig. 6 Graph of the proportion of receptors which are free, bound, internalized as a function of external LDL concentration in the steady-state where the total number of receptors is prescribed $(f=1$, $k_{r}=0$ ). Dashed lines correspond to the results of (Harwood and Pellarin, 1997): "- + - " indicates the proportion of bound receptors, ' $-*-$ ' indicates the proportion of internalized receptors, ' $-o-$ ' indicates the proportion of free receptors. Solid lines indicate the results from our model: ' + ' the proportion of bound receptors, ' $*$ ' the proportion of internalized receptors, ' $o$ ' the proportion of free receptors, the solid line with no additional marking shows the percentage occupancy of receptors on the surface (that is, bound/(bound+free)).

the loss of receptors due to imperfect recycling of internalized pits $(f<1)$ and de novo production of pits via the term $k_{r} /(K+C)$. Since this introduces cholesterol-dependence into the system, the resulting steady-state is more complex.

Here, we consider the case $\alpha \gg 1$ in which, to leading order, the extracellular LDL concentration does not vary. Thus, as in Section 4.1, we take $L_{e}$ as a constant, and calculate the steady-state to which the cellular variables $N_{0}, N, R, C, L_{i}, L_{b}$ tend. The steadystate is now given by

$$
\begin{aligned}
& 0=g R-A p_{m} L_{e} N_{0}-b_{0} N_{0}, \\
& 0=A p_{m} L_{e} N_{0}-N, \\
& 0=A L_{e}\left(p_{m} N+p_{m} N_{0}-M\right)-M, \\
& 0=\frac{k_{r}}{K+C}+f N+f b_{0} N_{0}-g R, \\
& 0=\psi M-k_{i d} L_{i}, \\
& 0=r k_{i d} L_{i}-\lambda(C-1),
\end{aligned}
$$




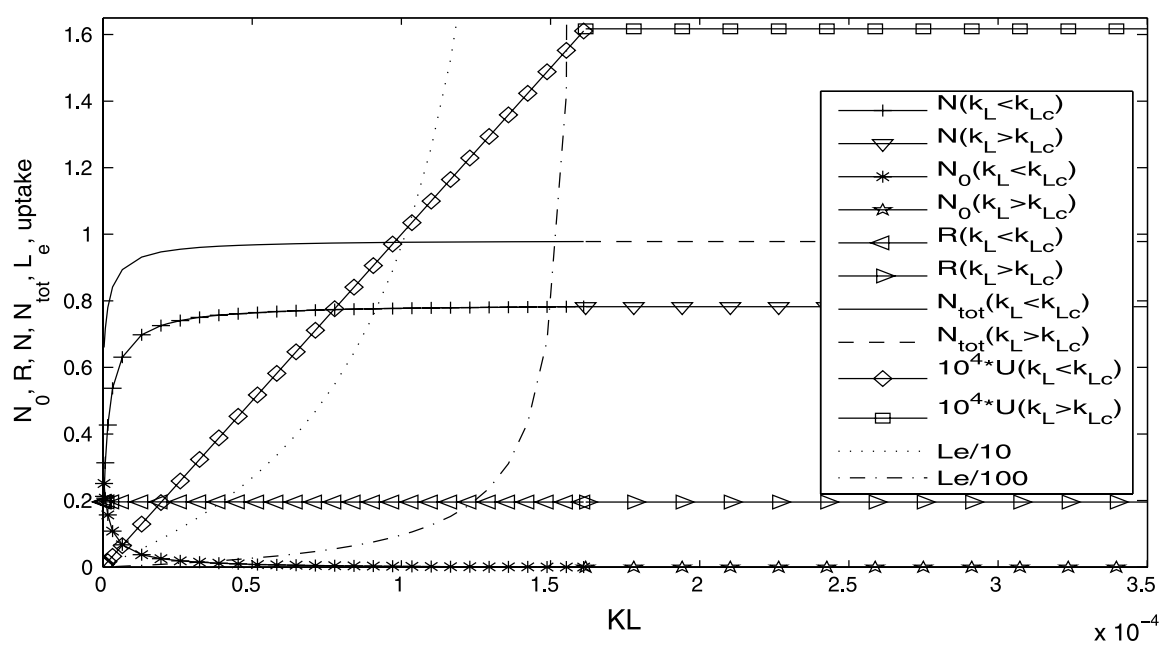

Fig. 7 Graphs of the steady-state number of occupied, empty and internalized pits $\left(N, N_{0}, R_{i}\right)$ as well as the total number of pits $\left(N_{\text {tot }}\right)$, LDL-uptake and $L_{e}$ plotted against the LDL delivery rate $k_{L}$ for $0<k_{L}<2.2 k_{L c}(82)$. The graph shows the change in these quantities across the range of steady-states $\left(k_{L}<k_{L c} \approx 1.7 \times 10^{-4}\right)$ and pseudo-steady states $\left(k_{L}>k_{L c} \approx 1.7 \times 10^{-4}\right)$ induced by the delivery rate $k_{L}$.

which can be solved in terms of $N_{0}$ by

$$
\begin{aligned}
N & =A p_{m} L_{e} N_{0}, \quad R=\frac{\left(b_{0}+A p_{m} L_{e}\right) N_{0}}{g}, \\
M & =\frac{A p_{m} L_{e}\left(1+A p_{m} L_{e}\right) N_{0}}{1+A L_{e}}, \\
L_{i} & =\frac{\psi A p_{m} L_{e}\left(1+A p_{m} L_{e}\right) N_{0}}{k_{i d}\left(1+A L_{e}\right)}, \quad C=1+\frac{r \psi A p_{m} L_{e}\left(1+A p_{m} L_{e}\right) N_{0}}{\lambda\left(1+A L_{e}\right)},
\end{aligned}
$$

wherein $N_{0}$ satisfies a quadratic equation and is given by

$$
\begin{aligned}
N_{0}= & \frac{\lambda\left(1+A L_{e}\right)(1+K)}{2 r \psi A p_{m} L_{e}\left(1+A p_{m} L_{e}\right)} \\
& \times\left[-1+\sqrt{1+\frac{4 r \psi A p_{m} L_{e} k_{r}\left(1+A p_{m} L_{e}\right)}{\lambda\left(1+A L_{e}\right)(1-f)(1+K)^{2}\left(b_{0}+A p_{m} L_{e}\right)}}\right] .
\end{aligned}
$$

Using the parameter values in Table 2, and for the values of $L_{e}$ that we are interested in, $A p_{m} L_{e} \gg 1$. Hence, the fraction inside the square root in (72) is small, the solution can be approximated by

$$
N_{0} \sim \frac{k_{r}}{(1-f)(1+K)\left(b_{0}+A p_{m} L_{e}\right)},
$$


and

$$
N \sim \frac{k_{r} A p_{m} L_{e}}{(1-f)(1+K)\left(b_{0}+A p_{m} L_{e}\right)}, \quad R \sim \frac{k_{r}}{g(1-f)(1+K)} .
$$

Using (74), we deduce further that the total number of pits $\left(N_{T}\right)$ in the steady-state

$$
N_{T}=\frac{k_{r}\left[\left(b_{0}+g\right)+(1+g) A p_{m} L_{e}\right]}{g(1+K)(1-f)\left(b_{0}+A p_{m} L_{e}\right)} \sim \frac{k_{r}(1+g)}{g(1+K)(1-f)} .
$$

As we might expect, $N_{T}$ is strongly dependent on the pit production term $k_{r} /(1+K)$ and on the recycling parameter $f$. However, $N_{T}$ changes very little with the external LDL concentration, dropping only at extremely low values of $L_{e}$. For the typical values of $L_{e}$ that we are concerned with, the $A p_{m} L_{e}$ terms dominate the brackets in the numerator and the denominator, leading to the approximation shown at the right of (75). The occupancy of pits in this case is exactly the same as that for the case $f=1, k_{r}=0$ (see Eq. (75) and Fig. 6).

Equation (75) gives a clear indication of whether there will be more or less pits at steady-state than at $t=0$. The condition for there to be fewer pits at steady-state $\left(N_{T}<1\right)$ is

$$
f<f_{c} \sim \frac{k_{r}(1+g)}{(1+K) g} .
$$

For the parameter values in Table 2, this gives a critical value of $f_{c}=0.9$.

\subsection{Steady-state for continuous delivery $\left(0<k_{L}<k_{L c}\right)$}

Including the input parameter $k_{L}>0$ adds to the complexity of the system (46)-(52) since now $L_{e}$ is unknown and must be determined as part of the solution.

By considering the sum $\psi \dot{M}+\alpha \dot{L}_{e}$, that is, Eqs. (48) and (50), as well as (51)-(52), we find that in the steady-state we have

$$
M=\frac{\alpha k_{L}}{\psi}, \quad L_{i}=\frac{\alpha k_{L}}{k_{i d}}, \quad C=1+\frac{r \alpha k_{L}}{\lambda} .
$$

The number of empty, occupied and internalized pits can then be calculated as

$$
\begin{aligned}
& N_{0}=\frac{\alpha k_{L}}{\psi A p_{m} L_{e}} \frac{\left(1+A L_{e}\right)}{\left(1+A p_{m} L_{e}\right)}, \quad N=\frac{\alpha k_{L}}{\psi} \frac{\left(1+A L_{e}\right)}{\left(1+A p_{m} L_{e}\right)}, \\
& R=\frac{\alpha k_{L}}{g \psi p_{m}} \frac{\left(1+A L_{e}\right)}{A L_{e}} \frac{\left(b_{0}+A p_{m} L_{e}\right)}{\left(1+A p_{m} L_{e}\right)} .
\end{aligned}
$$

This leaves a quadratic for the extracellular concentration

$$
\Omega\left(1+A L_{e}\right)\left(b_{0}+A p_{m} L_{e}\right)=A L_{e}\left(1+A p_{m} L_{e}\right),
$$

where

$$
\Omega=\frac{(1-f) \alpha k_{L}}{\psi k_{r} p_{m}}\left(K+1+\frac{r \alpha k_{L}}{\lambda}\right),
$$


is a measure of the input strength which is more convenient than $k_{L}$. Note that this is quadratic in $k_{L}$, which means that we expect small values of $k_{L}$ to make little difference to the results already found for the case $k_{L}=0$, but that as larger values of $k_{L}$ are considered, we expect to observe more rapid change in behavior.

Given the parameters in Table 2 and any positive value of $k_{L}$, the solution of (79) always has real roots. For $0<\Omega<1$, the roots have opposite sign, the physically relevant one being that which gives a positive value for $L_{e}$, namely

$$
L_{e}=\frac{1}{2 A p_{m}(1-\Omega)}\left[b_{0} \Omega+p_{m} \Omega-1+\sqrt{\left(b_{0} \Omega+p_{m} \Omega-1\right)^{2}+4 b_{0} p_{m} \Omega(1-\Omega)}\right] .
$$

As $\Omega$ rises from zero, $L_{e}$ also increases monotonically from zero, and as $\Omega \rightarrow 1^{-}, L_{e}$ becomes arbitrarily large. For $\Omega>1$, both roots for $L_{e}$ are negative, and hence unphysical; this case is analyzed in the next subsection, see Section 4.4 for details.

The critical value $\Omega=1$ corresponds to a critical input rate $k_{L c}$ obtained by inverting the relationship (80)

$$
k_{L}=k_{L c}:=\frac{\lambda}{2 \alpha r}\left[\sqrt{(K+1)^{2}+\frac{4 r \psi p_{m} k_{r}}{\lambda(1-f)}}-K-1\right] .
$$

Thus, for $0<k_{L}<k_{L c}$, the system approaches a steady-state. For $k_{L}$ close to $k_{L c}$, this state has a large extracellular concentration of $\operatorname{LDL}\left(L_{e} \gg 1\right)$. Results from this section are illustrated in Fig. 7, however, since this figure includes the results of Section 4.4, we delay discussion of the figure until the end of that section.

\subsection{Pseudo-steady-state for continuous delivery $\left(k_{L}>k_{L c}\right)$}

As noted above, for $k_{L}>k_{L c}$, there is no physically-relevant steady-state solution because the rate of delivery of LDL is too large for the cell to internalize all that arrives at its surface. Hence, there is a state in which the extracellular concentration grows linearly in time (and can become unboundedly large), whilst the cell variables approach a steadystate. We will refer to this situation as a pseudo-steady-state.

To find the behavior when $k_{L}>k_{L c}$, we first consider the form of the steady-state solution in the limit $k_{L} \rightarrow k_{L c}^{-}$. In this limit $\Omega \rightarrow 1^{-}, L_{e} \rightarrow+\infty$ and Eq. (78) implies

$$
N_{0} \rightarrow 0, \quad N \sim \frac{\alpha k_{L c}}{\psi p_{m}}, \quad M \sim \frac{\alpha k_{L c}}{\psi}, \quad R \sim \frac{\alpha k_{L c}}{g \psi p_{m}} .
$$

Hence, in this limit pits are full, $M /\left(N+N_{0}\right) \rightarrow p_{m}$. We expect this behavior to carry over into the case $k_{L}>k_{L c}$.

The steady-state solution for $k_{L}<k_{L c}$ suggests that the cell can process LDL particles at some rate which is less than or equal to $k_{L c}$, and the excess LDL accumulates in the extracellular medium. Hence, we seek a large-time asymptotic solution of the form

$$
\begin{aligned}
L_{e} & \sim \Lambda t+\widehat{L}, & N_{0} & \sim \frac{v}{t}, \quad N \sim N_{1}+\frac{N_{2}}{t}, \quad M \sim M_{1}+\frac{M_{2}}{t}, \\
R & \sim R_{1}+\frac{R_{2}}{t}, & L_{i} & \sim L_{1}+\frac{L_{2}}{t}, \quad C \sim C_{1}+\frac{C_{2}}{t}, \quad \text { as } t \rightarrow \infty,
\end{aligned}
$$


where $v$ has been introduced as a coefficient in the rate at which the concentration of empty pits decays to zero. Although this could be viewed as a correction term to the leading order solution $N_{0}=0, v$ influences directly the leading-order behavior of many of the concentrations.

Equations (47), (46), (48), (51), and (52) imply, respectively,

$$
\left.\begin{array}{l}
N_{1}=A p_{m} \Lambda \nu, \quad R_{1}=\frac{A p_{m} \Lambda v}{g}, \quad M_{1}=A p_{m}^{2} \Lambda v, \\
L_{1}=\frac{A p_{m}^{2} \Lambda v \psi}{k_{i d}}, \quad C_{1}=1+\frac{A p_{m}^{2} \Lambda v r \psi}{\lambda}, \quad p N_{2}-M_{2}=\left(p_{m}-1\right) p_{m} \nu .
\end{array}\right\}
$$

The final equation comes from the first-correction terms of (48). In (85), the constants $\Lambda$ and $v$ are determined by substitution from (85) in (49) and (50):

$$
\frac{k_{r}}{1-f}=N_{1}\left(K+1+\frac{r \psi p_{m} N_{1}}{\lambda}\right), \quad \alpha \Lambda=\alpha k_{L}-\psi p_{m} N_{1} .
$$

Derivation of the latter equation relies on knowledge of the higher order correction terms $p N_{2}-M_{2}$ given in (85). The former equation has a unique solution in $N_{1}>0$, the latter gives $\Lambda$, as follows

$$
\begin{aligned}
& N_{1}=\frac{\lambda(K+1)}{2 r \psi p_{m}}\left[\sqrt{1+\frac{4 k_{r} p_{m} r \psi}{\lambda(1-f)(K+1)^{2}}}-1\right], \\
& \Lambda=k_{L}-\frac{\psi p_{m} N_{1}}{\alpha}, \quad v=\frac{N_{1}}{A p_{m} \Lambda} .
\end{aligned}
$$

We remark that $N_{1}$ does not depend on $k_{L}$ or $\Lambda$. Consequently, as the LDL input rate increases $\left(k_{L}\right)$, the number of occupied pits remains constant whereas the rate of increase in the concentration of the extracellular LDL increases linearly with $k_{L}$ and the rate at which empty pits decrease over time diminishes. The equations for $\Lambda$ and $v$; however, do depend on the LDL input rate, $k_{L}$, so the rate of increase of external LDL, $L_{e}$, and the rate at which empty pits diminish in number will also depend on the input rate.

Figure 7 shows how the behavior of the system changes as $k_{L}$ ranges from zero to $2.2 k_{L c}$ in the case where $k_{L c}=1.6 \times 10^{-4}$. Nondimensional steady-states are plotted against $k_{L}$. The proportion of empty pits drops from 0.25 to almost zero as $k_{L}$ increases from zero to $\frac{1}{5} k_{L c}$, the steady-state is precisely zero for $k_{L}>k_{L c}$. As $k_{L}$ rises from zero to $0.3 \times 10^{-4}=\frac{1}{5} k_{L c}$, the number of occupied pits rapidly rises from zero to approximately 0.75 . If we consider larger values of $k_{L}$, from $\frac{1}{5} k_{L c}$ to $2.2 k_{L c}$, there is very little further increase in the number of occupied pits $(N)$. There is no perceptible change in the number of any type of pits in the range $\frac{1}{5} k_{L c}<k_{L}<2.2 k_{L c}$; and almost no change in the number of internalized pits across the whole range of $k_{L}$. Such quantities show no abrupt changes in the behavior of the cell in the cases $k_{L}<k_{L c}$ and $k_{L}>k_{L c}$, rather, there is an abrupt change in the range $0<k_{L}<\frac{1}{5} k_{L c}$, then a plateau is reached which shows little variation for $k_{L}>\frac{1}{5} k_{L c}$. The cell's internal cholesterol level rises linearly from unity at $k_{L}=0$ to $C=1.003$ at $k_{L}=k_{L c}$ and then stays at 1.003 for $k_{L}>k_{L c}$. The quantities which do show abrupt changes are the pit occupancy, which rises linearly from zero to $100 \%$ in the range 
$0<k_{L}<k_{L c}$, the external LDL concentration $L_{e}$ and the uptake (the last two of which are plotted in Fig. 7).

We might expect the steady increase in $L_{e}$ and the resulting decrease to zero of $N_{0}$ to dramatically alter the cell's regulation of pit production. However, since intracellular cholesterol levels do not rise significantly, there is no significant increase or reduction in pit production or rate of internalization of LDL particles. The cell reaches a maximum rate of uptake of LDL and simply keeps operating at that level.

\section{Discussion}

We have proposed a model of LDL particle adhesion to clathrin-coated pits on the surface of hepatocytes. Whilst this process has been modeled before, we believe that this is the first model to take account of the structure of receptors being grouped into pits of approximately 200 receptors which are all internalized simultaneously to form a lysosome. Furthermore, our model includes the breakdown of these internalized structures, the recycling of a proportion of the receptors, and the regulation of new receptor-production by the cell's internal cholesterol concentration.

Following the construction of a detailed microscopic model, we reduce the system of over 200 ordinary differential equations (Sections 2.1-2.2) to just seven (Section 2.3). Parameters are found from the literature (2.4), the model is nondimensionalized (Section 2.5) and verified against the experimental results of Brown and Goldstein (1979) (Section 3.1) and the model of Harwood and Pellarin (1997) (Section 4.1). Here, we show that the model correctly predicts the proportions of free, bound, and internalized receptors, as a function of external LDL concentration, assuming the system is at steady-state.

As well as presenting our best-fit to the results of Brown and Goldstein (1979) (Fig. 2), Section 3 contains details of the evolution of all the other (nonmeasurable) variables in the system. We also summarize the results of a detailed sensitivity analysis in which every parameter in the model has been varied, and its influence on all variables measured. Those parameters which have the dominant influence have been highlighted and discussed. The sensitivity coefficient of bound labeled LDL (denoted $L_{b l}$ in this paper) relative to the rate of endocytosis $(\bar{b})$, has been found to be an order of magnitude larger than all other sensitivity coefficients. This is an elegant quantitative illustration of the appropriateness of the Brown and Goldstein experimental setup (Brown and Goldstein, 1979) to the investigation of endocytosis processes, as the rate of disappearance of the LDL label is highly sensitive to the rate of receptor mediated endocytosis $(\bar{b})$ while at the same time robust to variation in all other parameters in the model. We have explored the effect of the recycling fidelity parameter $f$ on the kinetics of the endocytosis process. This system provides a good example of the tradeoff between a robust, responsive system which rapidly equilibrates, but leads to less efficient use of pits at low values of $f$, and a more efficient process which takes longer to reach steady-state at higher values of $f$.

Elevated LDL-C levels have been shown to play a role in the development and progression of CHD. In particular, individuals with familial hypercholesterolaemia $(\mathrm{FH})$ have been shown to have circulating LDL-C concentrations that are much higher than normal levels, which can be caused by defects in the LDL-R, the binding protein apo B-100, or more recently discovered protein involved in the degradation of the LDLreceptors, PCSK9 (Abifadel et al., 2003). The effects of these varying mechanisms 
can be explored using our model by varying the parameters associated with binding $\left(\bar{A}, p_{m}\right)$, or intracellular recycling of receptors and pits $(f)$ (Zhang et al., 2007; Lagace et al., 2006).

In Section 4, we also use the model to speculate on the form of solution in the case where LDL particles are added to the extracellular medium at a constant rate. The system will then approach a steady-state in which LDL particles are constantly being taken into the cell, broken down, most receptors being recycled, and new ones being manufactured, replacing the nonrecycled ones, so that the total number of receptors is dependent on the cell's internal cholesterol level. Such a situation can only be maintained if the rate of delivery of LDL is below a critical threshold value $\left(k_{L}<k_{L c}\right)$. For delivery rates above this value, the system approaches a pseudo-steady state in which the extracellular LDL concentration grows linearly with time, the number of empty pits decreases to zero, the number of free receptors also approaches zero and the cell is at steady-state of maximum LDL processing capacity. This pseudo-steady state is very similar to the exact steady-states for delivery rates below critical value $\left(\frac{1}{5} k_{L c}<k_{L}<k_{L c}\right)$. The main change in behavior of the cell occurring in the low LDL delivery rates of $0<k_{L}<\frac{1}{5} k_{L c}$.

\section{Acknowledgements}

We are grateful to Pieter de Groot for making helpful comments on the manuscript, also to Marcus Tindall for many useful discussions. JADW thanks Unilever for hospitality. We acknowledge financial support from the EPSRC for funding an Springboard fellowship for JADW [grant number EP/E032362/1], and for supporting the (2005) Mathematics-inMedicine study group where this problem was first analyzed (Panovska et al., 2006).

Open Access This article is distributed under the terms of the Creative Commons Attribution Noncommercial License which permits any noncommercial use, distribution, and reproduction in any medium, provided the original author(s) and source are credited.

\section{References}

Abifadel, M., Varret, M., Rabès, J.P., Allard, D., Ouguerram, K., Devillers, M., Cruaud, C., Benjannet, S., Wickham, L., Erlich, D., Derré, A., Villéger, L., Farnier, M., Beucler, I., Bruckert, E., Chambaz, J., Chanu, B., Lecerf, J.M., Luc, G., Moulin, P., Weissenbach, J., Prat, A., Krempf, M., Junien, C., Seidah, N.G., Boileau, C., 2003. Mutations in PCSK9 cause autosomal dominant hypercholesterolemia. Nat. Genet. 34, 154-156.

Aravindhan, K., Webb, C.L., Jaye, M., Ghosh, A., Willette, R.N., DiNardo, N.J., Jucker, B.M., 2006. Assessing the effects of LXR agonists on cellular cholesterol handling: a stable isotope tracer study. J. Lipid Res. 47, 1250-1260.

August, E., Parker, K.H., Barahona, M., 2007. A dynamical model of lipoprotein metabolism. Bull. Math. Biol. 69, 1233-1254. Also available at arXiv.org/abs/q-bio/0610053.

Basu, S.K., 1984. Receptor-mediated endocytosis: an overview of a dynamic process. J. Biosci. 6, 535542.

Basu, S.K., Goldstein, J.L., Brown, M.S., 1978. Characterization of the low density lipoprotein receptor in membranes prepared from human fibroblasts. J. BioPhys. Chem. 253, 3852-3856.

Bradley, W.A., Huang, S.-L.C., Karlin, J.B., Lin, A.H.Y., Prasad, S.C., Gotto, A.M. Jr., Gianturco, S.H., 1984. Low-density lipoprotein receptor binding determinants switch from apolipoprotein E to apolipoprotein B during conversion of hypertriglyceridemic very-low density lipoprotein to lowdensity lipoproteins. J. Biol. Chem. 259, 14728-14735. 
Brown, M.S., Goldstein, J.L., 1979. Receptor-mediated endocytosis: insights from the lipoprotein receptor system. Proc. Natl. Acad. Sci. USA 76, 3330-3337.

Cho, B.H.S., Choue Dokko, R., Hong Chung, B., 2002. Oleic, linoleic and linolenic acids enhance receptor-mediated uptake of low density lipoproteins in Hep-G2 cells. J. Nut. Biochem. 13, 330-336.

Chun, P.W., Espinosa, A.J., Lee, C.W., Shireman, R.B., Brumbaugh, E.E., 1985. Low density lipoprotein receptor regulation. Biophys. Chem. 21, 185-196.

Dunn, K.W., McGraw, T.E., Maxfield, F.R., 1989. Iterative fractionation of recycling receptors from lysosomally destined ligands in an early sorting endosome. J. Cell Biol. 109, 3303-3314.

Galeano, N.F., Al-Haideri, M., Keyserman, F., Rumsey, S.C., Deckelbaum, R.J., 1998. Small dense low density lipoprotein has increased affinity for LDL receptor-independent cell surface binding sites: a potential mechanism for increased atherogenicity. J. Lipid Res. 39, 1263-1273.

Goldstein, J.L., Anderson, R.G.W., Brown, M.S., 1979. Coated pits, coated vesicles and receptor-mediated endocytosis. Nature 279, 679-685.

Goldstein, B., Wofsy, C., 1981. Analysis of coated pit recycling on human fibroblasts. Cell Biophys. 3, 251-277.

Harwood, H.J., Pellarin, L.D., 1997. Kinetics of low-density lipoprotein receptor activity in Hep-G2 cells: derivation and validation of a Briggs-Haldane-based kinetic model for evaluating receptor-mediated endocytotic processes in which receptors recycle. Biochem. J. 323, 649-659.

Jackson, K.G., 2005. Personal communication.

Jackson, K.G., Maitin, V., Leake, D.S., Yaqoob, P., Williams, C.M., 2006. Saturated fat-induced changes in $S_{f}$ 60-400 particle composition reduces uptake of LDL by HepG2 cells. J. Lipid Res. 47, 393-403.

Jackson, K.G., Wolstencroft, E.J., Bateman, P.A., Yaqoob, P., Williams, C.M., 2005. Greater enrichment of triacylglycerol-rich lipoproteins with apolipoproteins $\mathrm{E}$ and $\mathrm{C}$-III after meals rich in saturated fatty acids than after meals rich in unsaturated fatty acids. Am. J. Clin. Nutr. 81, 25-34.

Lagace, T.A., Curtis, D.E., Garuti, R., McNutt, M.C., Park, S.W., Prather, H.B., Anderson, N.N., Ho, Y.K., Hammer, R.E., Horton, J.D., 2006. Secreted PCSK9 decreases the number of LDL receptors in hepatocytes and in livers of parabiotic mice. J. Clin. Invest. 116, 2995-3005.

Mamotte, C.D.S., Sturm, M., Foo, J.I., van Bockxmeer, F.M., Taylor, R.R., 1999. Comparison of the LDLreceptor binding of VLDL and LDL from apoE4 and apoE3 homozygotes. Am. J. Physiol. 276 (Endocrinol. Metab. 39), E553-E557.

Panovska, J., Pickersgill, L., Tindall, M., Wattis, J.A.D., Byrne, H.M., 2006. Mathematical models of hepatic lipoprotein metabolism. Available from www2.maths.ox.ac.uk/ociam/Study-Groups/ MMSG05/reports/liverreport.pdf.

Pearson, T., Wattis, J.A.D., O’Malley, B., Pickersgill, L., Jackson, K.G., Byrne, H.M., 2008. Mathematical Modelling of competitive LDL and VLDL binding, and endocytosis by hepatocytes. J. Math. Biol. submitted.

Shankaran, H., Resat, H., Wiley, H.S., 2007. Cell surface receptors for signal transduction and ligand transport: a design principles study. PLoS Comput. Biol. 3, 0986-0999.

Tindall, M.J., Wattis, J.A.D., O’Malley, B., Pickersgill, L., Jackson, K.G., 2008. Mathematical model of lipoprotein metabolism, submitted.

Zhang, D.W., Lagace, T.A., Garuti, R., Zhao, Z., McDonald, M., Horton, J.D., Cohen, J.C., Hobbs, H.H., 2007. Binding of proprotein convertase subtilisin/kexin type 9 to epidermal growth factor-like repeat A of low density lipoprotein receptor decreases receptor recycling and increases degradation. J. Biol. Chem. 282, 18602-18612. 